JOURNAL OF THE

AMERICAN MATHEMATICAL SOCIETY

Volume 24, Number 2, April 2011, Pages 567-601

S 0894-0347(2010)00686-8

Article electronically published on November 30, 2010

\title{
AUTOMORPHISMS OF MULTIPLICITY FREE HAMILTONIAN MANIFOLDS
}

\author{
FRIEDRICH KNOP
}

\section{INTRODUCTION}

Consider a connected compact Lie group $K$ acting on a connected Hamiltonian manifold $M$. A measure for the complexity of $M$ is half the dimension of the symplectic reductions of $M$, and it is natural to study Hamiltonian manifolds with low complexity first, starting with the case of complexity zero, the so-called multiplicity free manifolds (see [GS] or [MiFo]). It has been a longstanding problem to classify multiplicity free manifolds, and it is the purpose of this paper to complete this project.

More specifically, Delzant conjectured in 1989 that any compact multiplicity free space is uniquely determined by two invariants: its momentum polytope $\mathcal{P}$ and its principal isotropy group $L_{0}$. Evidence for this conjecture was Delzant's celebrated classification of multiplicity free torus actions [De1, as well as further particular cases settled by Iglésias $(K=S O(3),[\operatorname{Igl}])$, Delzant ( $r k K=2, \overline{D e} 2)$, and Woodward (transversal actions, Wo1). The main objective of this paper is, building upon work of Losev [Los, to complete the proof of Delzant's conjecture (see Theorem 10.2).

Once we know that a multiplicity free manifold is characterized by the combinatorial data $\left(\mathcal{P}, L_{0}\right)$ it is natural to ask which pairs actually arise this way. In section 11 we show that this can be reduced to a purely local problem on $\mathcal{P}$. More precisely, $\mathcal{P}$ has to "look" locally like the weight monoid of a smooth affine spherical variety (see Theorem 11.2). Since the latter class of varieties has been previously classified by Van Steirteghem and the author [KVS this finishes the classification of multiplicity free manifolds.

The proof of the Delzant Conjecture proceeds in two separate steps: first a local statement (ultimately due to Losev [Los]) and then a local-to-global argument (addressed in this paper).

First, we describe briefly the local problem. Let $\mathfrak{t} \subseteq \mathfrak{k}$ be a Cartan subalgebra. Then it is well known that the orbit space $\mathfrak{k}^{*} / K$ can be identified with a Weyl chamber $\mathfrak{t}^{+} \subseteq \mathfrak{t}$. Thus the moment map $m: M \rightarrow \mathfrak{k}^{*}$ gives rise to the invariant moment map $\psi: M \rightarrow \mathfrak{t}^{+}$. By a celebrated theorem of Kirwan Kir1, the image $\mathcal{P}=$ $\psi(M)$ is a convex polytope for $M$ compact. The local statement asserts now that two compact multiplicity free manifolds with the same momentum polytope $\mathcal{P}$ and the same principal isotropy group are isomorphic locally over $\mathcal{P}$ (see Theorem 2.4). Using techniques from $\mathrm{Sja}$, one can reduce this local problem to a statement about

Received by the editors August 18, 2010 and, in revised form, October 1, 2010

2010 Mathematics Subject Classification. Primary 53D20, 14L30, 14M27.

(C) 2010 by Friedrich Knop 
smooth affine spherical varieties, the "Knop Conjecture" (see Theorem 2.7), which was recently settled affirmatively by Losev [Los.

To pass from local to global, we need to determine the automorphism group of a multiplicity free manifold. More precisely, we need to know the sheaf of automorphisms $\mathcal{A}_{M}$ of $M$ over $\mathcal{P}$. Our main result is (see section [10):

1.1. Theorem. Let $M$ be a compact multiplicity free manifold with moment polyhedron $\mathcal{P}$. Then $\mathcal{A}_{M}$ is a sheaf of abelian groups. Moreover, all of its higher cohomology groups vanish: $H^{i}\left(\mathcal{P}, \mathcal{A}_{M}\right)=0$ for $i \geq 1$.

As an application, the vanishing of $H^{1}$ implies that two compact multiplicity free manifolds which are locally isomorphic over $\mathcal{P}$ are isomorphic globally. Together with the local statement this yields the Delzant Conjecture. Moreover, the vanishing of $H^{2}$ implies that there are no obstructions for gluing manifolds which are given locally over $\mathcal{P}$ to one global manifold $M$.

The bulk of this paper is devoted to computing the sheaf $\mathcal{A}_{M}$. More precisely, we show that $\mathcal{A}_{M}$ is controlled by a certain root system $\Phi_{M}$. In a sense, this root system is a symplectic analogue to the restricted root system of a symmetric space and is just as fundamental to understanding the geometry of a multiplicity free manifold.

The main technique of our approach rests on the fact that multiplicity free manifolds are modeled locally by smooth affine spherical varieties. This idea is not new; e.g., Sjamaar Sja uses it to reprove Kirwan's convexity theorem. But instead of (locally) embedding $M$ into a smooth affine spherical variety $X$ as an open subset, we embed $M$ into the cotangent bundle $T_{X}^{*}$ as a closed totally real subset. This way, one can think of $T_{X}^{*}$ as a complexification of $M$. The geometry of these cotangent bundles has been the focus of much of our previous research. In particular, invariants and the automorphisms have been worked out in detail in [Kn1 and [Kn3], respectively. Thus, the main task solved in this paper is to port these algebraic results to the smooth category.

As a preliminary step for determining all automorphisms we have to first study $K$-invariant smooth functions on $M$ since these generate automorphisms via Hamiltonian flows. It will turn out (see Theorem 4.1) that the smooth $K$-invariants are controlled by a finite reflection group $W_{M}$. This group is then used to construct the root system $\Phi_{M}$. Note that this part of the paper is a specialization of our paper [Kn5] where manifolds of arbitrary complexity were considered. We decided not to refer to that paper, though, since some arguments simplify dramatically in the multiplicity free case.

We conclude this introduction with some historical remarks. The local-to-global principle, Theorem 1.1, was proved by us around 1995, but circumstances prevented our publishing a formal proof until now. Around the same time we announced the local statement, Theorem 2.4, or more precisely its algebraic equivalent (Theorem 2.7) as a conjecture (see e.g. Kn4). In joint work with Bart Van Steirteghem, we worked out a classification $\mathrm{KVS}$ of smooth affine spherical varieties in an attempt to prove the conjecture. Unfortunately, this turned out to be unfeasible due to the multitude of cases to consider. Meanwhile (around 2000), Luna launched a program to classify all spherical varieties and completed it for groups of type A Lun. This enabled his student Camus Cam to settle the "Knop Conjecture" for groups of type A. Finally, Losev [Los managed to bypass all problems which still exist in Luna's program and proved the conjecture in full generality. This 
event reinvigorated our interest in the subject so that finally the proof of Delzant's conjecture was completely documented.

Notation. In the following, $K$ is a compact connected Lie group with Lie algebra $\mathfrak{k}$. Let $T_{\mathbb{R}} \subseteq K$ be a maximal torus with Lie algebra $\mathfrak{t}_{\mathbb{R}}$ and Weyl group $W$. We fix moreover a Weyl chamber $\mathfrak{t}^{+} \subseteq \mathfrak{t}_{\mathbb{R}}^{*}$.

Complexifications: For any compact Lie group $H$, we denote its complexification by $H^{c}$. In particular, we put $G=K^{c}$, a connected reductive complex algebraic group. Then $T:=T_{\mathbb{R}}^{c}$ is a maximal torus of $G$ with Lie algebra $\mathfrak{t}=\mathfrak{t}_{\mathbb{R}}^{c}$. Let $\Lambda_{T}:=\operatorname{Hom}\left(T, \mathbb{C}^{\times}\right)$be the character group of $T$. Then $T=\operatorname{Hom}\left(\Lambda_{T}, \mathbb{C}^{\times}\right)$with $\mathbb{R}$-structure

$$
\chi(\bar{t})=\overline{\chi(t)}^{-1} .
$$

This way, the group of real points $T(\mathbb{R})$ coincides with the compact torus $T_{\mathbb{R}}$. Likewise, $t^{*}=\Lambda \otimes_{\mathbb{Z}} \mathbb{R}$ carries the real structure

$$
\overline{\chi \otimes z}:=-\chi \otimes \bar{z}
$$

such that $\mathfrak{t}_{\mathbb{R}}^{*}$ is identified with

$$
\left\{a \in \mathfrak{t}^{*} \mid \bar{a}=a\right\}=\Lambda_{\mathbb{Z}} \otimes i \mathbb{R} .
$$

\section{The local Delzant conjecture}

Besides developing notation which is used throughout this paper, we describe in this section how to reduce the local problem mentioned in the introduction to the purely algebraic statement solved by Losev.

Let $M$ be a (possibly non-compact) connected Hamiltonian $K$-manifold with moment map $m: M \rightarrow \mathfrak{k}^{*}$. The momentum image of $M$ is the set $\mathcal{P}=m(M) \cap \mathfrak{t}^{+}$. A theorem of Kirwan Kir1 states that $\mathcal{P}$ is a convex polyhedron when $M$ is compact. There is another way to look at it: The restriction of the quotient map $\mathfrak{k}^{*} \rightarrow \mathfrak{k}^{*} / K$ to $\mathfrak{t}^{+}$is a homeomorphism $\pi_{+}: \mathfrak{t}^{+} \rightarrow \mathfrak{k}^{*} / K$. Now we define the invariant moment map as the composed map

$$
\psi: M \stackrel{m}{\rightarrow} \mathfrak{k}^{*} \rightarrow \mathfrak{k}^{*} / K \stackrel{\pi_{+}^{-1}}{\longrightarrow} \mathfrak{t}^{+} .
$$

It is important to keep in mind that $\psi$ is, in general, not differentiable. The polytope $\mathcal{P}$ is then the image of $\psi$.

2.1. Definition. A Hamiltonian $K$-manifold $M$ is multiplicity free if it is connected and if $\operatorname{dim} M / K=\operatorname{dim} \mathcal{P}$, i.e., if $\psi: M \rightarrow \mathcal{P}$ has discrete fibers.

If $M$ is compact, then (by another theorem of Kirwan, see Kir2]) all fibers of $\psi$ are connected. Thus, $M$ is multiplicity free if and only if $\psi$ is a topological quotient.

In our approach it is necessary to also consider non-compact manifolds. More specifically, let $U \subseteq \mathcal{P}$ be open. Then $M_{U}:=\psi^{-1}(U)$ is, in general, a non-compact multiplicity free manifold, and we need to determine its automorphism group, as well. For arbitrary non-compact multiplicity free manifolds certain pathologies may occur, such as $M / K \rightarrow \mathcal{P}$ having non-connected fibers or being bijective but not a homeomorphism. Thus we restrict our attention to convex manifolds in the sense 
of [Kn6]. For multiplicity free manifolds the definition boils down to:

2.2. Definition. A multiplicity free manifold is convex if

i) the momentum image $\mathcal{P}$ is convex and

ii) the invariant moment map $\psi: M \rightarrow \mathcal{P}$ is proper.

Under these conditions, one can show that $M / K \rightarrow \mathcal{P}$ is a homeomorphism and that $\mathcal{P}$ is locally polyhedral, i.e., for every $a \in \mathcal{P}$ there is a polyhedral cone $C_{a} \subseteq \mathfrak{t}_{\mathbb{R}}^{*}$ and an open neighborhood $U$ of $a$ in $\mathfrak{t}_{\mathbb{R}}^{*}$ such that

$$
\mathcal{P} \cap U=\left(a+C_{a}\right) \cap U .
$$

In particular, $\mathcal{P}$ is locally closed.

The restriction to convexity is quite mild. Clearly, compact Hamiltonian manifolds are convex. Moreover, every multiplicity free manifold is, at least, locally convex. So, our theory applies to some extent even to the general case. For the remainder of this section we will assume $M$ to be convex and multiplicity free.

We proceed by recalling some facts about the principal isotropy group. Let $\mathfrak{a}^{0} \subseteq \mathfrak{t}_{\mathbb{R}}^{*}$ be the affine subspace spanned by $\mathcal{P}$. The interior of $\mathcal{P}$ inside $\mathfrak{a}^{0}$ is called its relative interior $\mathcal{P}^{0}$. It is open and dense in $\mathcal{P}$. The centralizer $L_{\mathbb{R}}$ of $\mathfrak{a}^{0}$ (or, equivalently, $\mathcal{P}$ ) is a Levi subgroup of $K$ containing the maximal torus $T$. The generic structure of $M$ is then described by the following well-known

\subsection{Lemma. Let $\Sigma:=m^{-1}\left(\mathcal{P}^{0}\right)$. Then:}

i) $\Sigma$ is a Hamiltonian $L_{\mathbb{R}}$-manifold with moment map $\left.m\right|_{\Sigma}$.

ii) Let $L_{0} \subseteq L_{\mathbb{R}}$ be the kernel of the action of $L_{\mathbb{R}}$ on $\Sigma$. Then $A_{\mathbb{R}}:=L_{\mathbb{R}} / L_{0}$ is a torus acting freely on $\Sigma$.

iii) The map $K \times{ }^{L_{\mathbb{R}}} \Sigma \rightarrow M$ is an open immersion. Thus, $L_{0}$ is a principal isotropy group for $K$ acting on $M$.

iv) The complement $M \backslash K \Sigma$ has codimension $\geq 2$. In particular, $K \Sigma$ is dense and $\Sigma$ is connected.

Proof. Let $F$ be the smallest face of the Weyl chamber $\mathfrak{t}^{+}$which contains $\mathcal{P}$ and let $F^{0}$ be its relative interior. Then $\mathcal{P}^{1}:=\mathcal{P} \cap F^{0}$ is open in $\mathcal{P}$ and contains $\mathcal{P}^{0}$. Let $\Sigma^{1}:=m^{-1}\left(\mathcal{P}^{1}\right)$. If $\Sigma$ is replaced by $\Sigma^{1}$, then all assertions iv iv except for the freeness of the $A$-action (which is false on $\Sigma^{1}$ ) have been shown in, e.g., LMTW (even for non-multiplicity free manifolds). Now observe that $\Sigma^{1}$ is a convex multiplicity free $A_{\mathbb{R}}$-manifold with momentum image $\mathcal{P}^{1}$. These actions have been studied by Delzant De1, and it follows from his theory that the $A_{\mathbb{R} \text {-action is free }}$ over $\mathcal{P}^{0}$ and that the points $x \in \Sigma^{1}$ with $m(x) \in \partial \mathcal{P}^{1}$ have an infinite isotropy group. Since $\Sigma^{1} \rightarrow \mathcal{P}^{1}$ is the quotient by $A_{\mathbb{R}}$, we conclude that the preimage of $\partial \mathcal{P}^{1}$ in $\Sigma^{1}$ has codimension $\geq 2$.

Remark. It is possible to show that $K \Sigma$ is exactly the union of all $K$-orbits of maximal dimension. In particular, it coincides with the open stratum of $M$.

Let $\mathfrak{a}_{\mathbb{R}}^{*}$ be the Lie algebra of $A_{\mathbb{R}}$. Then the momentum image of $\Sigma$ is an open subset in a translate of $\mathfrak{a}_{\mathbb{R}}^{*}$ in $\mathfrak{t}_{\mathbb{R}}^{*}$. This implies that $\mathfrak{a}_{\mathbb{R}}^{*}$ is in turn the linear subspace of $\mathfrak{t}_{\mathbb{R}}^{*}$ which is parallel to the affine space $\mathfrak{a}^{0}$. From that it follows that the momentum image $\mathcal{P}$ alone determines the Lie algebras $\mathfrak{l}_{\mathbb{R}}$ and $\mathfrak{l}_{0}$ of $L_{\mathbb{R}}$ and $L_{0}$, respectively: $\mathfrak{l}_{\mathbb{R}}$ is the centralizer of $\mathcal{P}$ and $\mathfrak{l}_{0}$ is the set of $\xi \in \mathfrak{l}_{\mathbb{R}}$ with $\chi_{1}(\xi)=\chi_{2}(\xi)$ for all $\chi_{1}, \chi_{2} \in \mathcal{P}$. 
In other words, the only additional information gained from $L_{0}$ is in the form of the group $\Lambda_{M}:=\operatorname{Hom}\left(A_{\mathbb{R}}, \mathbb{C}^{\times}\right)$considered as a lattice in $i \mathfrak{a}_{\mathbb{R}}^{*} \subseteq \mathfrak{a}_{\mathbb{R}}^{*} \otimes_{\mathbb{R}} \mathbb{C}$.

Now we state the local statement mentioned in the introduction. Recall that $M_{U}=\psi^{-1}(U)$.

2.4. Theorem. For $\nu \in\{1,2\}$ let $M_{\nu}$ be a convex multiplicity free Hamiltonian $K$-manifold with invariant moment map $\psi_{\nu}$. Assume $\psi_{1}\left(M_{1}\right)=\psi_{2}\left(M_{2}\right)=: \mathcal{P}$ and $L_{0}\left(M_{1}\right)=L_{0}\left(M_{2}\right)$. Then every $a \in \mathcal{P}$ has a (convex) open neighborhood $U$ such that $\left(M_{1}\right)_{U} \cong\left(M_{2}\right)_{U}$ as a Hamiltonian K-manifold.

First we reduce to the case $a=0$. Let $\mathfrak{t}_{a}^{+}$be the open star of $\mathfrak{t}^{+}$in $a$, i.e., $\mathfrak{t}^{+}$ with all faces removed which do not contain $a$. Let $L_{\mathbb{R}} \subseteq K$ be the centralizer of $a$. Then $\mathfrak{l}_{a}^{+}:=\operatorname{Ad} L_{\mathbb{R}}\left(\mathfrak{t}_{a}^{+}\right)$is a connected open subset of $\mathfrak{l}_{\mathbb{R}}^{*}$. Put $\Sigma_{\nu}:=m_{\nu}^{-1}\left(\mathfrak{l}_{a}^{+}\right)$. The cross-section theorem (see e.g. GSj], 2.4) asserts that $K \times{ }^{L_{\mathbb{R}}} \Sigma_{\nu} \rightarrow M_{\nu}$ is an open embedding of Hamiltonian manifolds. It follows that the momentum images of $M_{\nu}$ and $\Sigma_{\nu}$ (with respect to $K$ and $L_{\mathbb{R}}$, respectively) are equal in a neighborhood of $a$. Moreover the principal isotropy group $L_{0}$ does not change. We may replace therefore $M_{\nu}$ by $\Sigma_{\nu}$. Since $a$ is a character of $\mathfrak{l}_{\mathbb{R}}$ we may replace the moment map $m_{\Sigma_{\nu}}$ of $\Sigma_{\nu}$ by its translate $m_{\Sigma_{\nu}}-a$. Thus, we may assume from now on that $a=0$.

Let, for the moment, $M$ be any convex Hamiltonian manifold and let $x \in M$ be a point with $m(x)=0$. Then the symplectic slice theorem (aka. equivariant Darboux theorem; see GSj, 2.3) asserts that a neighborhood of $K x$ in $M$ is uniquely determined by two data: the isotropy group $H_{\mathbb{R}}:=K_{x}$ and the symplectic slice $S=(\mathfrak{k} x)^{\perp} /(\mathfrak{k} x)$, which is a symplectic representation of $H_{\mathbb{R}}$. Here, $\mathfrak{k} x$ is the tangent space of the orbit $K x$ in $x$.

It is well known that every symplectic representation of a compact group carries a compatible unitary structure, i.e., such that the symplectic form is the imaginary part of the Hermitian form. Thus the action of $H_{\mathbb{R}}$ extends to an action of its complexification $H$. Now put $X:=G \times{ }^{H} S$ (recall $G=K^{c}$ ) which is a smooth affine complex algebraic $G$-variety.

To relate $X$ with $M$ we equip $X$ with the structure of a Hamiltonian $K$-manifold: first embed $X$ equivariantly into a finite-dimensional $G$-module $V$ and choose a $K$-invariant Hermitian scalar product on $V$. Then $X$ inherits the structure of a Hamiltonian $K$-manifold from $V$.

2.5. Lemma. The orbits $K x \subseteq M$ and $K / H_{\mathbb{R}} \subseteq G / H \subseteq X$ have convex open $K$ invariant neighborhoods which are isomorphic as Hamiltonian $K$-manifolds. Moreover, the momentum images of $M, X$ and the open subsets agree in a neighborhood of 0 .

Proof. It is easy to see that the orbits $K x$ and $K / H_{\mathbb{R}}$ share the same local data $\left(H_{\mathbb{R}}, S\right)$. So the existence of isomorphic open sets follows from the symplectic slice theorem. Moreover, if $U \subseteq M$ is open and $K$-invariant, then $\psi(U)$ is an open subset of $\mathcal{P}$. This implies the second assertion.

The lemma allows us to replace $M$ by $X$. Recall that $X$ is spherical if a Borel subgroup of $G$ has a dense open orbit. This is equivalent to the ring of functions $\mathbb{C}[X]$ being a multiplicity free $G$-module. Its structure, as a $G$-module, is determined by the set $\Xi_{X}$ of highest weights (the so-called weight monoid). Now, we 
have the following comparison theorem due to Brion [Bri]:

2.6. Theorem. Let $X=G \times{ }^{H} S$ be as above. Then

i) $X$ is a multiplicity free $K$-manifold if and only if $X$ is a spherical $G$-variety.

ii) Let $\mathcal{P}$ be the momentum image of $X$ and let $\mathcal{Q}$ be the convex cone generated by $\Xi_{X}$. Then $\mathcal{P}=i \mathcal{Q}$.

iii) Let $\Lambda_{X}$ be the lattice determining the principal isotropy group $L_{0}$ of $X$. Then $\Lambda_{X}=\left\langle\Xi_{X}\right\rangle_{\mathbb{Z}}$.

iv) Conversely, $\Xi_{X}=\mathcal{Q} \cap \Lambda_{X}$.

Now we deduce the local Delzant conjecture from the following theorem. It was conjectured by the author around 1996 (see, e.g., [Kn4]) and proved by Losev [Los] in 2007.

2.7. Theorem. Two smooth affine spherical $G$-varieties with the same weight monoid are G-equivariantly isomorphic.

Proof of Theorem 2.4. As explained above, we may assume $a=0$. Likewise, we may replace $M_{\nu}$ by $X_{\nu}=G \times{ }^{H^{\nu}} S_{\nu}$, where $H_{\mathbb{R}}^{\nu} \subseteq K$ is a closed subgroup and $S_{\nu}$ is a unitary representation of $H_{\mathbb{R}}^{\nu}$. Part iv) of Theorem 2.6 implies that $X_{1}$ and $X_{2}$ have the same weight monoid. Thus, Theorem 2.7 provides a $G$-equivariant isomorphism $\varphi: X_{1} \stackrel{\sim}{\rightarrow} X_{2}$.

The variety $X_{\nu}$ contains a unique closed $G$-orbit, namely $G / H^{\nu}$. Thus $\varphi$ induces an isomorphism $G / H^{1} \stackrel{\sim}{\rightarrow} G / H^{2}$. Let $\varphi\left(e H^{1}\right)=g_{0} H^{2}$ with $g_{0} \in G$. Then $H^{2}=$ $g_{0}^{-1} H^{1} g_{0}$.

Let $\mathfrak{k}=\mathfrak{h}_{\mathbb{R}}^{\nu} \oplus \mathfrak{p}_{\nu}$ be an $H_{\mathbb{R}}^{\nu}$-invariant decomposition. Then it is well known that the map

$$
K \times^{H_{\mathbb{R}}^{\nu}} \mathfrak{p}_{\nu} \rightarrow G / H^{\nu}:[k, \xi] \mapsto k \exp (i \xi)
$$

is an isomorphism of $K$-manifolds. It follows that, as a $K$-manifold, $G / H^{\nu}$ has a single minimal $K$-stratum, namely $K / H_{\mathbb{R}}^{\nu} \times \exp \left(i\left(\mathfrak{p}_{\nu}^{H_{\mathbb{R}}^{\nu}}\right)\right)$. The isomorphism $\varphi$ maps the minimal stratum to a minimal stratum. Thus, we get the decomposition $g_{0}=c k$ with $k \in K$ and $H_{\mathbb{R}}^{2}=k^{-1} H_{\mathbb{R}}^{1} k$ and where $c=\exp (i \xi) \in G$ centralizes $H_{\mathbb{R}}^{2}$.

Now we change the isomorphism $\varphi$ by the automorphism of $X_{2}=G \times{ }^{H^{2}} S_{2}$ which maps $[g, s]$ to $\left[g c^{-1}, s\right]$. Thereby we achieve $c=1$ and therefore $g_{0}=k \in K$. By replacing $x_{2}$ with $k x_{2}$ we even get $g_{0}=k=1$. Then $H^{1}=H^{2}$. The isomorphism $S_{1} \cong S_{2}$ follows since $S_{\nu}$ is the complex normal space of $G / H^{\nu} \subseteq X_{\nu}$ in $x_{\nu}$.

\section{THE LOCAL MODEL AND ITS INVARIANTS}

Let $M$ be a Hamiltonian manifold and $x \in M$ a point with $m(x)=0$. The slice theorem asserts that a neighborhood of $K x$ is determined by two data: the isotropy group $H_{\mathbb{R}}=K_{x}$ and the space $S=(\mathfrak{k} x)^{\perp} /(\mathfrak{k} x)$, considered as a symplectic representation of $H_{\mathbb{R}}$. Let $G$ and $H$ be the complexifications of $K$ and $H_{\mathbb{R}}$, respectively. In the previous section, we worked with the local model $X=G \times{ }^{H} S$, but from now on it is more convenient to use

$$
\mathrm{M}:=K \times^{H_{\mathbb{R}}}\left(\mathfrak{h}_{\mathbb{R}}^{\perp} \oplus S\right) .
$$

Since $K, H_{\mathbb{R}}$, and $S$ are real algebraic varieties, the same holds for $\mathrm{M}$. Let $\mathrm{M}^{c}:=$ 
Spec $\mathbb{C}[M]$ be the complexification of $\mathrm{M}$. This variety is related to $X$ in the following way:

3.1. Theorem. Let $\mathrm{M}, \mathrm{M}^{c}$, and $X$ be as above. Then

i) $\mathrm{M}^{c}$ is isomorphic to the cotangent bundle $T_{X}^{*}$ of $X$ as a Hamiltonian $G$ variety. In particular, the diagram of moment maps commutes:

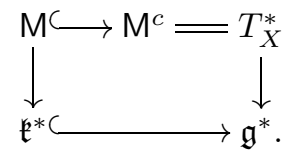

ii) $\mathrm{M}$ is multiplicity free if and only if $X$ is spherical.

Proof. Let $\langle\cdot, \cdot\rangle$ denote the $H_{\mathbb{R}}$-invariant Hermitian scalar product on $S$. Then $S$ is a Hamiltonian manifold with symplectic form $\omega_{S}\left(s_{1}, s_{2}\right)=\operatorname{Im}\left\langle s_{1}, s_{2}\right\rangle$ and moment $\operatorname{map} m_{S}(s)=\left(\xi \in \mathfrak{h}_{\mathbb{R}} \mapsto \frac{1}{2} \omega_{S}(\xi s, s)\right)$. Thus also

$$
Z_{\mathbb{R}}:=T_{K}^{*} \times S=K \times \mathfrak{k}^{*} \times S
$$

is a symplectic $H_{\mathbb{R}}$-manifold with moment map

$$
m(k, \kappa, s)=-\left.\kappa\right|_{\mathfrak{h}_{\mathbb{R}}}+m_{S}(s) .
$$

After choosing an $H_{\mathbb{R}}$-stable complement $\mathfrak{p}_{\mathbb{R}}$ of $\mathfrak{h}_{\mathbb{R}}$ in $\mathfrak{k}$ one can extend the linear form $m_{S}(s) \in \mathfrak{h}_{\mathbb{R}}^{*}$ to the linear form $\ell_{s} \in \mathfrak{k}^{*}$ with $\ell_{s}(\mathfrak{p})=0$. Then

$$
K \times \mathfrak{h}_{\mathbb{R}}^{\perp} \times S \stackrel{\sim}{\rightarrow} m^{-1}(0):(k, \kappa, s) \mapsto\left(k, \ell_{s}+\kappa, s\right) .
$$

After taking the quotient by $H_{\mathbb{R}}$ we see that $\mathrm{M}$ is isomorphic to the symplectic reduction of $Z_{\mathbb{R}}$ in $0 \in \mathfrak{k}^{*}$.

Now we complexify. The Hermitian scalar product yields an isomorphism

$$
\bar{S} \stackrel{\sim}{\rightarrow} S^{*}: s \mapsto \frac{1}{2 i}\langle\cdot, s\rangle .
$$

Then $S \hookrightarrow S \otimes_{\mathbb{R}} \mathbb{C}=S \oplus \bar{S}=S \oplus S^{*}$, and a short calculation shows that the restriction of the canonical symplectic form on $S \oplus S^{*}=T_{S}^{*}$ to $S$ is the standard form $\omega_{S}=\operatorname{Im}\langle\cdot, \cdot\rangle$. Thus, the embedding of $Z_{\mathbb{R}}$ into its complexification

$$
Z=G \times \mathfrak{g}^{*} \times S \times S^{*}=T_{G \times S}^{*}
$$

is compatible with the Hamiltonian structure. Recall that the symplectic reduction in 0 of a cotangent bundle is the cotangent bundle of the quotient. Thus, the symplectic reduction of $Z$ is $T_{X}^{*}$, showing $i$ ). Finally, ii) is just Theorem [2.6 in conjunction with Lemma 2.5.

Remark. Observe that, even though $T_{X}^{*}$ is defined over $\mathbb{R}$ as a Hamiltonian variety, neither is $X$ nor the projection $T_{X}^{*} \rightarrow X$.

The $G$-invariants on $T_{X}^{*}$ have been determined in [Kn1]. We proceed by summarizing the most important facts.

3.2. Theorem. Let $X$ be a smooth affine spherical variety. Let $\Lambda_{X} \subseteq \mathfrak{t}^{*}$ be the subgroup generated by the weight monoid and let $\mathfrak{a}^{*}$ be its $\mathbb{C}$-span. Then there is a finite subgroup $W_{X} \subseteq G L\left(\mathfrak{a}^{*}\right)$ and a morphism $q: T_{X}^{*} \rightarrow \mathfrak{a}^{*} / W_{X}$ with:

i) $q$ is the categorical quotient of $T_{X}^{*}$ by $G$.

ii) $W_{X}$ is generated by reflections. In particular, $\mathfrak{a}^{*} / W_{X}$ is isomorphic to an affine space. 
iii) $W_{X}$ is a subgroup of $N_{W}\left(\mathfrak{a}^{*}\right) / C_{W}\left(\mathfrak{a}^{*}\right)$ and normalizes the lattice $\Lambda_{X} \subseteq \mathfrak{a}^{*}$.

Proof. This is [Kn1, Satz 6.4, Satz 6.6 and [Kn2, Lemma 3.4, Theorem 4.2.

To apply this theorem to $\mathrm{M}$, recall that $\mathfrak{a}^{*}=\Lambda_{X} \otimes_{\mathbb{Z}} \mathbb{C}$ has the real structure

$$
\overline{\chi \otimes z}=-\chi \otimes \bar{z} .
$$

Thus, the real points are $\mathfrak{a}_{\mathbb{R}}^{*}=\Lambda_{X} \otimes i \mathbb{R}$. Recall (Theorem 2.6) that $\psi: \mathrm{M} \rightarrow \mathfrak{t}^{+}$ takes values in $\mathfrak{a}_{\mathbb{R}}^{*}$.

For any $n \in N_{W}\left(\mathfrak{a}^{*}\right)$ put ${ }^{n} W_{X}:=n W_{X} n^{-1}$. Then there is an isomorphism

$$
\tilde{n}: \mathfrak{a}^{*} / W_{X} \stackrel{\sim}{\longrightarrow} \mathfrak{a}^{*} /{ }^{n} W_{X}: W_{X} a \mapsto{ }^{n} W_{X} n a .
$$

3.3. Theorem. Assume that $\mathrm{M}$ is multiplicity free. Then there is $n \in N_{W}\left(\mathfrak{a}^{*}\right)$ such that the following diagram commutes:

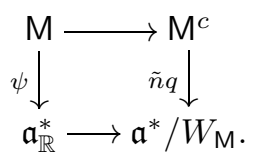

Here $W_{\mathrm{M}}:={ }^{n} W_{X}$.

Proof. Consider the fiber product

$$
\mathrm{M}_{N}^{c}:=\mathrm{M}^{c} \times_{\mathfrak{a}^{*} / N} \mathfrak{a}^{*},
$$

where $N=N_{W}\left(\mathfrak{a}^{*}\right) / C_{W}\left(\mathfrak{a}^{*}\right)$. This variety is in general not irreducible, and one of its irreducible components is

$$
\mathrm{M}_{e}^{c}:=\mathrm{M}^{c} \times_{\mathfrak{a}^{*} / W_{X}} \mathfrak{a}^{*}
$$

(see [Kn2], Lemma 3.4). The others are translates of $\mathrm{M}_{e}^{c}$ by an element $n \in N$ :

$$
\mathrm{M}_{n}^{c}:=\mathrm{M}^{c} \times_{\mathfrak{a}^{*} / W_{X}}^{n} \mathfrak{a}^{*},
$$

where the superscript ${ }^{n}$ indicates that the map $\mathfrak{a}^{*} \rightarrow \mathfrak{a}^{*} / W_{X}$ is twisted by $\mathfrak{a}^{*} \stackrel{n}{\rightarrow} \mathfrak{a}^{*}$.

The morphism $\mathfrak{a}^{*} / N \rightarrow \mathfrak{t}^{*} / W$ is finite and birational onto its image. Thus $\mathfrak{a}^{*} / N$ is the normalization of the image of $\mathfrak{a}^{*}$ in $\mathfrak{t}^{*} / W$. The compatibility of moment maps (3.2) yields therefore the commutative diagram

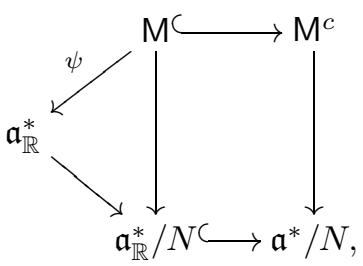

hence a map

$$
\varphi: \mathrm{M} \rightarrow \mathrm{M} \times_{\mathfrak{a}_{\mathbb{R}}^{*} / N} \mathfrak{a}_{\mathbb{R}}^{*} \hookrightarrow \mathrm{M}^{c} \times_{\mathfrak{a}^{*} / N} \mathfrak{a}^{*} .
$$

Because $\psi$ is not smooth, we cannot expect $\varphi$ to be smooth. But if we restrict to the dense open subset $\mathrm{M}^{0}:=K \Sigma=K m^{-1}\left(\mathcal{P}^{0}\right)$ as in Lemma 2.3. then $\psi$ and $\varphi$ become even real analytic. Since $\mathrm{M}^{0}$ is connected, we conclude that $\varphi$ maps $\mathrm{M}^{0}$ 
into a single irreducible component $\mathrm{M}_{n}^{c}$ for some $n \in N$. By continuity, we obtain $\varphi(\mathrm{M}) \subseteq \mathrm{M}_{n}^{c}$. This means that the diagram

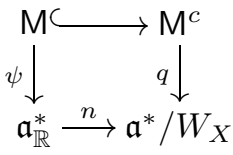

commutes. This is easily seen to be equivalent to the commutativity of (3.10).

Remark. The appearance of $n$ is unavoidable at this stage since we didn't specify how $q$ was defined. It is very likely, however, that $n$ is always 1 . With the methods of Wo2 it would be possible to show that $n$ normalizes $W_{X}$, i.e., that $W_{\mathrm{M}}=W_{X}$.

Now we use the preceding theory to determine the smooth $K$-invariants on M.

3.4. Corollary. The composed map $\psi / W_{\mathrm{M}}: \mathrm{M} \stackrel{\psi}{\rightarrow} \mathfrak{a}_{\mathbb{R}}^{*} \rightarrow \mathfrak{a}_{\mathbb{R}}^{*} / W_{\mathrm{M}}$ is smooth and

$$
\left(\psi / W_{\mathbf{M}}\right)^{*}: \mathcal{C}^{\infty}\left(\mathfrak{a}_{\mathbb{R}}^{*}\right)^{W_{\mathrm{M}}} \rightarrow \mathcal{C}^{\infty}(\mathbf{M})^{K}
$$

is surjective.

Proof. The map $\mathrm{M} \rightarrow \mathfrak{a}_{\mathbb{R}}^{*} / W_{\mathrm{M}}$ is a quotient by $K$ in the algebraic category. A theorem of Schwarz $\mathrm{Sch}$ asserts that then

$$
\mathcal{C}^{\infty}\left(\mathfrak{a}_{\mathbb{R}}^{*} / W_{\mathrm{M}}\right) \rightarrow \mathcal{C}^{\infty}(\mathrm{M})^{K}
$$

is surjective. The same theorem applied to $W_{\mathrm{M}}$ shows that $\mathcal{C}^{\infty}\left(\mathfrak{a}_{\mathbb{R}}^{*} / W_{\mathrm{M}}\right)$ $=\mathcal{C}^{\infty}\left(\mathfrak{a}_{\mathbb{R}}^{*}\right)^{W_{\mathrm{M}}}$.

Since $M$ is just a local model we are going to need a local refinement. Let $U \subseteq \mathfrak{a}_{\mathbb{R}}^{*}$ be a subset which we do not assume to be $W_{\mathrm{M}}$-invariant. Then, in abuse of language, we denote the image of $U$ in $\mathfrak{a}_{\mathbb{R}}^{*} / W_{\mathrm{M}}$ by $U / W_{\mathrm{M}}$. It carries an induced differentiable structure: a function on $U / W_{\mathrm{M}}$ is smooth if it is locally the restriction of a smooth function on $\mathfrak{a}_{\mathbb{R}}^{*} / W_{\mathrm{M}}$.

3.5. Corollary. For an open subset $U$ of $\mathcal{P}$, the momentum image of $\mathrm{M}$, let $\mathrm{M}_{U}:=$ $\psi^{-1}(U) \subseteq$ M. Then

$$
\mathcal{C}^{\infty}\left(U / W_{\mathrm{M}}\right) \stackrel{\sim}{\rightarrow} \mathcal{C}^{\infty}\left(\mathrm{M}_{U}\right)^{K} .
$$

Proof. Injectivity is clear. To prove surjectivity, let $f$ be some $K$-invariant on M. A partition of unity argument shows that we may assume that $f$ has compact support. But then it extends to all of $\mathrm{M}$ by zero and we conclude with Corollary 3.4

\section{INVARIANTS OF MULTIPLICITY FREE MANIFOLDS}

In this section, we determine the smooth invariants on an arbitrary convex multiplicity free manifold.

4.1. Theorem. Let $M$ be a convex multiplicity free Hamiltonian manifold with momentum image $\mathcal{P}=\psi(M)$. Let $\mathfrak{a}^{0} \subseteq \mathfrak{t}_{\mathbb{R}}^{*}$ be the affine space spanned by $\mathcal{P}$. Then:

i) There is a finite group $W_{0} \subseteq N_{W}\left(\mathfrak{a}^{0}\right) / C_{W}\left(\mathfrak{a}^{0}\right)$ such that the composed map $\psi / W_{0}: M \rightarrow \mathfrak{a}^{0} / W_{0}$ is smooth and the induced map

$$
\left(\psi / W_{0}\right)^{*}: \mathcal{C}^{\infty}\left(\mathcal{P} / W_{0}\right) \stackrel{\sim}{\rightarrow} \mathcal{C}^{\infty}(M)^{K}
$$

is an isomorphism. 
ii) Among all groups $W_{0}$ as in i), there is a unique minimal one, denoted by $W_{M}$. It is characterized by the fact that it is generated by reflections which have a fixed point in $\mathcal{P}$.

Remarks. 1. It is easy to construct an example where $\mathfrak{t}_{\mathbb{R}}^{*}=\mathbb{R}, \mathfrak{t}^{+}=\mathbb{R}_{\geq 0}, W=$ $\{ \pm 1\}$ and $\mathcal{P} \subseteq \mathfrak{t}^{+}$is a closed interval not containing the origin (take e.g. $K=$ $S U(2)$ acting on $\mathbb{P}^{2}(\mathbb{C})$ blown up at one point). Then the map $\mathcal{P} \rightarrow \mathbb{R} /\{ \pm 1\}$ is a diffeomorphism onto its image. Thus both $W_{0}=1$ and $W_{0}=\{ \pm 1\}$ would work. In particular, the group $W_{0}$ as in $i$ ) is not unique.

2. Let $\tilde{W} \subseteq N_{W}\left(\mathfrak{a}^{0}\right) / C_{W}\left(\mathfrak{a}^{0}\right)$ be the subgroup generated by all reflections which have a fixed point in $\mathcal{P}$. Then $W_{M} \subseteq \tilde{W}$ with equality being the rule rather than the exception. The standard example for when $W_{M} \subsetneq \tilde{W}$ is $K=S U(2)$ acting on $M=\mathbb{C}^{2}$. In fact, in that case the moment map is homogeneous of degree 2 and the generating invariant on $\mathfrak{k}^{*}$ is of degree 2 as well. So, the minimal degree of an invariant which is a pull-back from $\mathfrak{t}^{*} / W$ is 4 . Thus, the degree 2 invariant $q(z)=\|z\|^{2}$ on $M$ cannot be a pull-back. This shows that $W_{M}=1$, whereas $\tilde{W}=W=\mathbb{Z} / 2 \mathbb{Z}$.

3. The theorem generalizes to arbitrary convex Hamiltonian $K$-manifolds if one replaces the ring of $K$-invariants in (4.1) by its Poisson center. See Kn5 for details.

First, we prove a local version of the theorem.

4.2. Lemma. For every $a \in \mathcal{P}$ there is a unique subgroup $W(a)$ of $N_{W}\left(\mathfrak{a}^{0}\right) / C_{W}\left(\mathfrak{a}^{0}\right)$ having a as a fixed point and an open convex neighborhood $U$ of $a$ in $\mathcal{P}$ such that

$$
\mathcal{C}^{\infty}(U / W(a)) \stackrel{\sim}{\rightarrow} \mathcal{C}^{\infty}\left(M_{U}\right)^{K},
$$

where $M_{U}=\psi^{-1}(U)$. Moreover, $W(a)$ is generated by reflections.

Proof. We first establish the existence of $W(a)$ and $U$. Let $K x=\psi^{-1}(a)$. If $a=0$, then the assertion follows from Corollary 3.5 and the fact that $M$ is isomorphic to some model $\mathrm{M}$ near $K x$. In this case $W(a)=W_{\mathrm{M}}$.

If $a \neq 0$ but $a \in\left(\mathfrak{k}^{*}\right)^{K}$, then we can shift the moment map by $-a$. Since $a$ is also fixed by $W$ it follows that $N_{W}\left(\mathfrak{a}^{*}\right) / C_{W}\left(\mathfrak{a}^{*}\right)=N_{W}\left(\mathfrak{a}^{0}\right) / C_{W}\left(\mathfrak{a}^{0}\right)$. Thus, we are reduced to the case $a=0$.

In general, we apply the cross-section theorem. It states that a neighborhood $M_{0}$ of $K x$ in $M$ is isomorphic to $K \times^{L} \Sigma$, where $L=K_{a}$ and $\Sigma$ is a multiplicity free $L$-manifold. Moreover, the momentum images of $M_{0}$ and $\Sigma$ coincide and form an open neighborhood of $a$ in $\mathcal{P}$. Because of $\mathcal{C}^{\infty}\left(K \times^{L} \Sigma\right)^{K}=\mathcal{C}^{\infty}(\Sigma)^{L}$ we may replace $M$ by $\Sigma$. Thus we are reduced to the case $a \in\left(\mathfrak{k}^{*}\right)^{K}$.

For the uniqueness part, let $W_{1}$ and $W_{2}$ be two groups having all advertised properties. Then there is a neighborhood $U$ of $a$ in $\mathcal{P}$ with

$$
\mathcal{C}^{\infty}\left(U / W_{1}\right)=\mathcal{C}^{\infty}\left(M_{U}\right)^{K}=\mathcal{C}^{\infty}\left(U / W_{2}\right) .
$$

Let $\mathbb{R} \llbracket \mathfrak{a}^{0} \rrbracket$ be the ring of formal power series in $a$. Then (4.3) implies that

$$
\mathbb{R} \llbracket \mathfrak{a}^{0} \rrbracket^{W_{1}}=\mathbb{R} \llbracket \mathfrak{a}^{0} \rrbracket^{W_{2}}
$$

(here we use $W_{\nu} a=a$ for this to make sense). But this implies that $W_{1}=W_{2}$ (look at fields of fractions and apply Galois theory). 
To glue the local Weyl groups $W(a)$ to a global one we need the following coherence property:

4.3. Lemma. Every $a \in \mathcal{P}$ has a neighborhood $U \subseteq \mathcal{P}$ with

$$
W(b)=W(a)_{b} \quad \text { for all } b \in U .
$$

Proof. Note that $\mathfrak{a}^{0} / W(a)_{b} \rightarrow \mathfrak{a}^{0} / W(a)$ is an isomorphism near $b$. Thus $W(a)_{b}=$ $W(b)$ by uniqueness of $W(b)$.

Next we prove a criterion for gluing all local groups $W(a)$ together:

4.4. Lemma. Let $\Delta$ be a finite root system and $\Delta^{+} \subseteq \Delta$ a system of positive roots. Let $\Sigma$ be a set of positive roots such that $\left\langle\alpha \mid \beta^{\vee}\right\rangle \leq 0$ for all $\alpha, \beta \in \Sigma, \alpha \neq \beta$. Then $\Sigma$ is the set of simple roots for a subroot system of $\Delta$.

Proof. Let $C$ be the $|\Sigma| \times|\Sigma|$-matrix whose entries are $\left\langle\alpha \mid \beta^{\vee}\right\rangle$ with $\alpha, \beta \in \Sigma$. Then $C=C^{\prime} D$, where $C^{\prime}$ is the Gram matrix $(\alpha, \beta)$ of $\Sigma$, hence positive semidefinite, and where $D$ is a diagonal matrix with the positive entries $2 /(\beta, \beta)$ on the diagonal. If $C$ were singular, then $\mathrm{Kac}$, Thm. 4.3 implies that there is a non-zero non-negative vector $v$ such that $C v=0$. In other words, 0 would be a positive linear combination of $\Sigma$ which is impossible since $\sigma \subseteq \Delta^{+}$. It follows that $C$ is a generalized Cartan matrix of finite type. Such a matrix is associated to a finite root system in a unique way. In particular, $\Sigma$ is its set of simple roots.

4.5. Lemma. There is a subgroup $W_{M}$ of $N:=N_{W}\left(\mathfrak{a}^{0}\right) / C_{W}\left(\mathfrak{a}^{0}\right)$ with $W(a)=$ $\left(W_{M}\right)_{a}$ for all $a \in \mathcal{P}$.

Proof. We claim that the group $W_{M}$ which is generated by $\bigcup_{a \in \mathcal{P}} W(a)$ has all the required properties. From $W_{M} \subseteq N$ it follows that $W_{M}$ is finite. Since each individual $W(a)$ is generated by reflections, $W$ is also generated by reflections. From $\mathcal{P} \subseteq \mathfrak{t}^{+}$it follows that $W_{x}=C_{W}\left(\mathfrak{a}^{0}\right)$ for all $x \in \mathcal{P}^{0}$. This means that every reflection hyperplane of $W_{M}$ meets $\mathcal{P}$ at most in its boundary. Hence, $\mathcal{P}$ is entirely contained in a single Weyl chamber, say $\mathfrak{a}^{+}$, of $W_{M}$.

The finiteness of $W_{M}$ also implies that it has a fixed point $a_{0} \in \mathfrak{a}^{0}$. Thus we may identify $\mathfrak{a}^{0}$ with $\mathfrak{a}_{\mathbb{R}}^{*}$ and assume that $W_{M}$ is acting linearly.

Since $N$ is a subquotient of $W$ and since $\mathfrak{a}_{\mathbb{R}}^{*}$ is defined over $\mathbb{Q}$ there is a lattice in $\mathfrak{a}_{\mathbb{R}}^{*}$ which is normalized by $W_{M}$. This means that $W_{M}$ is in fact a crystallographic reflection group, i.e., that it is the Weyl group attached to a root system $\Delta$.

Every $W(a)$ is the Weyl group of a subroot system $\Delta(a) \subseteq \Delta$. Moreover, the Weyl chamber $\mathfrak{a}^{+}$determines a Weyl chamber for $\Delta(a)$ and therefore a set $\Sigma(a) \subseteq$ $\Delta(a)$ of simple roots. Moreover, the coherence property Lemma 4.3 implies that

$$
\Sigma(b)=\{\gamma \in \Sigma(a) \mid \gamma(b)=0\}
$$

for all $a \in \mathcal{P}$ and all $b \in \mathcal{P}$ sufficiently close to $a$. This follows from the fact that the isotropy group $W(a)_{b}$ is generated by simple reflections if $b$ is in the dominant Weyl chamber.

Now let $\Sigma$ be the union of all $\Sigma(a), a \in \mathcal{P}$. We claim that $\Sigma(a)$ can be recovered from $\Sigma$, namely:

$$
\Sigma(a)=\{\alpha \in \Sigma \mid \alpha(a)=0\} .
$$

Indeed, (4.6) implies " $\subseteq$ ". Conversely, let $\alpha \in \Sigma$ with $\alpha(a)=0$. By construction, $\alpha \in \Sigma(b)$ for some $b \in \mathcal{P}$. Thus $\alpha(a)=\alpha(b)=0$ and therefore $\alpha(c)=0$ for all $c$ on 
the line segment $[a, b]$. The convexity of $\mathcal{P}$ implies $[a, b] \subseteq \mathcal{P}$. From (4.6) it follows that the set of $c \in[a, b]$ with $\alpha \in \Sigma(c)$ is both open and closed. Hence $\alpha \in \Sigma(a)$, proving the claim.

Next, we want to apply Lemma 4.4, For that let $\alpha \in \Sigma(a)$ and $\beta \in \Sigma(b)$ with $a, b \in \mathcal{P}$. Then $a, b \in \mathfrak{a}^{+}$implies $\alpha(b) \geq 0, \beta(a) \geq 0$. If both $\alpha(b)>0$ and $\beta(a)>0$, then $\gamma(a)<0$ and $\gamma(b)>0$, where $\gamma:=\alpha-\beta$. Thus $\gamma$ is not a root, which implies $\left\langle\alpha, \beta^{\vee}\right\rangle \leq 0$. On the other hand if $\alpha(b)=0$, then $\alpha, \beta \in \Sigma(b)$ by (4.7). Thus, again $\left\langle\alpha, \beta^{\vee}\right\rangle \leq 0$. The same conclusion holds for $\beta(a)=0$. Lemma 4.4 implies that $\Sigma$ is the set of simple roots for some subroot system of $\Delta$ and therefore of $\Delta$ itself since $W_{M}$ is generated by the reflections in $\Sigma$. It follows that $\left(W_{M}\right)_{a}$ is generated by the reflections corresponding to the roots $\alpha \in \Sigma$ with $\alpha(a)=0$. Thus $\left(W_{M}\right)_{a}=W(a)$ by (4.7).

Proof of Theorem 4.1. Put $W_{0}=W_{M}$ as in Lemma 4.5. Then $M \rightarrow \mathfrak{a}_{\mathbb{R}}^{*} / W_{M}$ is smooth since it is locally smooth. Moreover, by a partition of unity argument, Lemma 4.2 shows that the map (4.1) is surjective. Finally, $W_{M}$ is the minimal possibility for $W_{0}$ since $W_{0}$ has to contain all local Weyl groups $W(a)$.

We conclude this section with a general statement about smooth functions on $\mathcal{P} / W_{M}$ which might be useful for concrete calculations. More precisely, since $\mathcal{P} \rightarrow$ $\mathcal{P} / W_{M}$ is bijective, functions on $\mathcal{P}$ are the same as functions on $\mathcal{P} / W_{M}$. So it is interesting to characterize those functions on $\mathcal{P}$ which are smooth on $\mathcal{P} / W_{M}$.

4.6. Proposition. Let $f: \mathcal{P} \rightarrow \mathbb{R}$ be a function. Then the following properties are equivalent:

i) $f \in \mathcal{C}^{\infty}\left(\mathcal{P} / W_{M}\right)$.

ii) For every $a \in \mathcal{P}$ there is a $\left(W_{M}\right)_{a}$-invariant smooth function $\tilde{f}$ on $\mathfrak{a}^{*}$ such that $f$ and $\tilde{f}$ coincide in a neighborhood of $a$ in $\mathcal{P}$.

iii) For every $a \in \mathcal{P}$ the Taylor series of $f$ in a is $\left(W_{M}\right)_{a}$-invariant.

If $\mathcal{P}$ is closed in $\mathfrak{a}^{*}$, then these conditions are equivalent to

iv) $f$ is the restriction of a smooth $W$-invariant function on $\mathfrak{a}^{*}$.

v) Let $g_{1}, \ldots, g_{r}$ be generators of $\mathbb{R}\left[\mathfrak{a}^{*}\right]^{W_{M}}$. Then there is a smooth function $h\left(x_{1}, \ldots, x_{r}\right)$ with $f=h\left(g_{1}, \ldots, g_{r}\right)$ on $\mathcal{P}$.

Proof. The implications is $\Rightarrow$ ii) $\Rightarrow$ iii) are clear. The implication iii) $\Rightarrow$ i) is a corollary of a deep theorem of Bierstone-Milman (BiMi], Thm. 3.2). In the case that $\mathcal{P}$ is closed, the last two conditions clearly imply the first three. Moreover, $v) \Rightarrow i v$, For the implication $i) \Rightarrow v$ ) observe that $\left(g_{1}, \ldots, g_{r}\right)$ embeds $\mathfrak{a}^{*} / W_{M}$, hence also $\mathcal{P} / W_{M}$, into $\mathbb{R}^{r}$ as a closed semi-algebraic subset. Then e.g. a partition of unity argument shows that $f$ can be extended to a smooth function $h$ on $\mathbb{R}^{r}$.

\section{The GRoup SCHEME}

The automorphisms of the cotangent bundle $T_{X}^{*}$ of a smooth spherical variety have been studied in Kn3. There it was shown that they can be interpreted as the global sections of a certain abelian group scheme acting on $T_{X}^{*}$. Our program is to extend this theory to multiplicity free manifolds.

In the present section, we recall the definition and some of the fundamental properties of the group scheme. For this, let $\mathfrak{a}$ be any finite-dimensional $\mathbb{C}$-vector space and let $W \subseteq G L(\mathfrak{a})$ be a finite reflection group. We assume that $W$ normalizes a lattice $\Lambda \subseteq \mathfrak{a}^{*}$. This implies that $W$ acts on the torus $A:=\operatorname{Hom}\left(\Lambda, \mathbb{C}^{\times}\right)$. We 
have $X(A)=\Lambda$ and Lie $A=\mathfrak{a}$. Let $\mathfrak{s}:=\mathfrak{a}^{*} / W=\operatorname{Spec} S^{*}(\mathfrak{a})^{W}$ be the quotient with quotient morphism $\pi: \mathfrak{a}^{*} \rightarrow \mathfrak{s}$. It is well known that $\mathfrak{s}$ is isomorphic to an affine space and that $\pi$ is finite and faithfully flat.

For any $\mathfrak{s}$-scheme $Y$, let $Y_{\mathfrak{a}^{*}}$ be the pull-back scheme $Y \times_{\mathfrak{s}} \mathfrak{a}^{*}$. It carries a natural action of $W$, and we are interested in $W$-equivariant morphisms $Y_{\mathfrak{a}^{*}} \rightarrow A$.

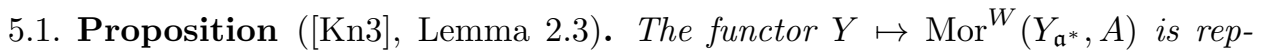
resented by a smooth affine abelian group scheme $\mathfrak{A}_{W} / \mathfrak{s}$. This means that a $W$ equivariant morphism $Y_{\mathfrak{a}^{*}} \rightarrow A$ is the "same" as a section of $\mathfrak{A}_{W}$ over $Y$, i.e., an $\mathfrak{s - m o r p h i s m ~} Y \rightarrow \mathfrak{A}_{W}$.

Example. Let $\mathfrak{a}=\mathbb{C}, W=\{ \pm 1\}$, and $\Lambda=\mathbb{Z}$. If $t$ is the coordinate function on $\mathfrak{a}^{*} \cong \mathbb{C}$, then $s=t^{2}$ is the coordinate function on $\mathfrak{s} \cong \mathbb{C}$. Let $\mathfrak{A} / \mathfrak{s}$ be the subgroup scheme of $G L(2, \mathbb{C}) \times \mathfrak{s}$ consisting of all pairs $(M, s)$ with

$$
M:=\left(\begin{array}{cc}
a & s b \\
b & a
\end{array}\right)
$$

and $\operatorname{det}(M)=a^{2}-s b^{2}=1$. We claim $\mathfrak{A}_{W} \cong \mathfrak{A}$. For this, we verify that $\mathfrak{A}$ has the required universal property. A morphism $Y \rightarrow \mathfrak{s}$ is given the image of $s$ in $\mathcal{O}(Y)$. Then every function on $Y_{\mathfrak{a}^{*}}$ has the form $a+t b$ with $a, b \in \mathcal{O}(Y)$ and $t^{2}=s$. A $W$-equivariant morphism $\varphi: Y_{\mathfrak{a}^{*}} \rightarrow A=\mathbb{C}^{\times}$amounts to an invertible function $a+t b$ with $(a+t b)^{-1}=a-t b$. Thus, $\varphi$ is equivalent to a pair $a, b \in \mathcal{O}(Y)$ with $a^{2}-s b^{2}=(a+t b)(a-t b)=1$, i.e., to an $\mathfrak{s}$-morphism $Y \rightarrow \mathfrak{A}$.

In the sequel, we need a refinement of $\mathfrak{A}_{W}$. In the example, observe that the generic fiber of $\mathfrak{A}$ is $\mathfrak{A}_{s} \cong \mathbb{C}^{\times}$while the zero fiber is $\mathfrak{A}_{0} \cong\{ \pm 1\} \times \mathbb{C}$. Thus, we obtain an open subgroup scheme $\mathfrak{A}^{\prime} \subset \mathfrak{A}$ by removing the set $\{-1\} \times \mathbb{C}$ inside $\mathfrak{A}_{0}$ from $\mathfrak{A}$. Since that set is a divisor, the group scheme $\mathfrak{A}^{\prime}$ is still affine.

In general, it was shown in Kn3 that the affine open subgroup schemes of $\mathfrak{A}_{W}$ correspond to the root systems with Weyl group $W$ and lattice $\Lambda$. More precisely, let $\Phi=\left(\Lambda, \Delta, \Lambda^{\vee}, \Delta^{\vee}\right)$ be a reduced root datum. Let $W=W(\Phi)$ be the Weyl group of $\Phi$ and let $A=\operatorname{Hom}\left(\Lambda, \mathbb{C}^{\times}\right)$be the torus with character group $\Lambda$. Then $\mathfrak{a}^{*}=\Lambda \otimes_{\mathbb{Z}} \mathbb{C}$.

For a root $\alpha \in \Delta$ let $s_{\alpha} \in W$ be the corresponding reflection. If we regard $\alpha$ as a character $A \rightarrow \mathbb{C}^{\times}$and the coroot $\alpha^{\vee}$ as a cocharacter $\mathbb{C}^{\times} \rightarrow A$, then the action of $s_{\alpha}$ on $A$ is given by the formula

$$
s_{\alpha}(t)=t \cdot \alpha^{\vee}(\alpha(t))^{-1} .
$$

Let $\chi \in \Lambda$ be an arbitrary character and let $t \in A$ with $s_{\alpha}(t)=t$. Then

$$
1=\chi\left(t \cdot s_{\alpha}(t)^{-1}\right)=\alpha(t)^{\left\langle\chi \mid \alpha^{\vee}\right\rangle} .
$$

The choice $\chi=\alpha$ implies in particular that $\alpha(t) \in\{1,-1\}$.

Now let $Z$ be a $W$-scheme and $Z^{s_{\alpha}}$ the $s_{\alpha}$-fixed point scheme. Let $\varphi: Z \rightarrow A$ be a $W$-equivariant morphism. Then the discussion shows $\alpha\left(\varphi\left(Z^{s_{\alpha}}\right)\right) \subseteq\{1,-1\}$.

5.2. Definition. A morphism $\varphi: Z \rightarrow A$ is called $\Phi$-equivariant if it is $W$ equivariant and if

$$
\alpha\left(\varphi\left(Z^{s_{\alpha}}\right)\right)=1
$$

for all roots $\alpha \in \Delta$. 
Remark. If there exists $\chi \in \Lambda$ with $\left\langle\chi \mid \alpha^{\vee}\right\rangle=1$, then (5.4) is automatically satisfied. This follows from (5.3). Most root systems have such a character $\chi$ (see Lemma 6.6 for details).

For a $W$-scheme $Z$, let $\operatorname{Mor}^{\Phi}(Z, A)$ be the set of all $\Phi$-equivariant morphisms $\varphi: Z \rightarrow A$. Again it was shown in [Kn3] that the functor $Y / \mathfrak{s} \mapsto \operatorname{Mor}^{\Phi}\left(Y_{\mathfrak{a}^{*}}, A\right)$ is represented by an affine commutative group scheme $\mathfrak{A}_{\Phi} / \mathfrak{s}$, i.e., with

$$
\operatorname{Mor}^{\Phi}\left(Y_{\mathfrak{a}^{*}}, A\right)=\operatorname{Mor}_{\mathfrak{s}}\left(Y, \mathfrak{A}_{\Phi}\right) .
$$

We proceed by summarizing some facts about $\mathfrak{A}=\mathfrak{A}_{\Phi}$. The proofs can be found either here or in Kn3].

Relation to $\mathfrak{A}_{W}$ : The group scheme $\mathfrak{A}_{\Phi}$ is an affine open subgroup scheme of $\mathfrak{A}_{W}$.

In fact, all affine open subgroup schemes are of this form. In particular, if $\Phi_{\max }$ is the root system where all roots are as long as possible, or equivalently where all coroots are primitive, then $\mathfrak{A}_{\Phi_{\max }}=\mathfrak{A}_{W}$.

Global sections: Let

$$
A^{\Phi}:=\{t \in A \mid \alpha(t)=1 \text { for all } \alpha \in \Delta\} .
$$

This is a subgroup of finite index of $A^{W}$, the group of $W$-fixed points in $A$. Every $t \in A^{\Phi}$ induces the constant $\Phi$-morphism $\mathfrak{a}^{*} \rightarrow A: \xi \mapsto t$ and therefore a section $\mathfrak{s} \rightarrow \mathfrak{A}$. In fact, since every morphism $\mathfrak{a}^{*} \rightarrow A$ is necessarily constant we have

$$
\Gamma(\mathfrak{s}, \mathfrak{A})=A^{\Phi} .
$$

Change of lattice: Let $\Lambda^{\prime} \subseteq \Lambda$ be a sublattice of finite index containing $\Delta$. Then $\Phi^{\prime}=\left(\Lambda^{\prime}, \Delta, \Lambda^{\prime \vee}, \Delta^{\vee}\right)$ is also a root datum and $A^{\prime}=\operatorname{Hom}\left(\Lambda^{\prime}, \mathbb{C}^{\times}\right)$is a quotient of $A$ by the finite group $E:=\operatorname{Hom}\left(\Lambda / \Lambda^{\prime}, \mathbb{C}^{\times}\right)$. Because of $\Delta \subseteq \Lambda^{\prime}$ we have $E \subseteq A^{\Phi}$. Thus $E$ acts (freely) on $\mathfrak{A}_{\Phi}$ by translation and $\mathfrak{A}_{\Phi} / E=\mathfrak{A}_{\Phi^{\prime}}$. In particular, the morphism $\mathfrak{A}_{\Phi} \rightarrow \mathfrak{A}_{\Phi^{\prime}}$ is finite and surjective.

Products of root system: Let $\Phi$ be the product of the root systems $\Phi_{1}$ and $\Phi_{2}$. Then $\mathfrak{a}^{*}=\mathfrak{a}_{1}^{*} \times \mathfrak{a}_{2}^{*}$ and $\mathfrak{A}_{\Phi}=\mathfrak{A}_{\Phi_{1}} \times \mathfrak{A}_{\Phi_{2}}$. For general root systems one can combine this with a suitable change of lattices as above. Thus there is always a finite quotient $\pi: \mathfrak{A} \rightarrow \mathfrak{A}^{\prime}$ or a covering $\pi: \mathfrak{A}^{\prime} \rightarrow \mathfrak{A}$ (with $\pi$ finite) where $\mathfrak{A}^{\prime}$ decomposes as

$$
\mathfrak{A}^{\prime}=\left(A_{0} \times \mathfrak{a}_{0}^{*}\right) \times \mathfrak{A}_{\Phi_{1}} \times \ldots \times \mathfrak{A}_{\Phi_{s}} .
$$

Here, $A_{0}$ is a torus with Lie algebra $\mathfrak{a}_{0}$ and the $\Phi_{\nu}$ are irreducible root systems.

Fibers: All fibers of $\mathfrak{A} / \mathfrak{s}$ are commutative of dimension $r=\operatorname{dim} A=\operatorname{rk} \Phi$. More precisely, for $a \in \mathfrak{a}^{*}$, let $s=\pi(a)$ be its image in $\mathfrak{s}$. Put

$$
\Delta_{a}:=\left\{\alpha \in \Delta \mid\left\langle a \mid \alpha^{\vee}\right\rangle=0\right\}
$$

and $\Phi_{a}=\left(\Lambda, \Delta_{a}, \Lambda^{\vee}, \Delta_{a}^{\vee}\right)$. Let $\mathfrak{A}_{s}=\mathfrak{A}_{\Phi} \times_{\mathfrak{s}}\{s\}$ be the fiber over $s$ and let $\mathfrak{A}_{s}=\mathfrak{A}_{s}^{\text {ss }} \times \mathfrak{A}_{s}^{\text {uni }}$ be its decomposition into a semisimple and a unipotent part. Then

$$
\mathfrak{A}_{s}^{\mathrm{ss}} \cong A^{\Phi_{a}} \subseteq A \quad \text { and } \quad \mathfrak{A}_{s}^{\mathrm{uni}} \cong \mathbb{C}^{\mathrm{rk}\left\langle\Delta_{a}\right\rangle}
$$

The first isomorphism depends on the choice of $a \in \pi^{-1}(s)$. 
Generic structure: A point $a \in \mathfrak{a}^{*}$ is called regular if its isotropy group $W_{a}$ is trivial. This condition is equivalent to $\Delta_{a}=\varnothing$, i.e., $\left\langle a \mid \alpha^{\vee}\right\rangle \neq 0$ for all $\alpha \in \Delta$. Let $\mathfrak{a}_{\text {reg }}^{*}$ be the set of regular points and let $\mathfrak{s}_{\text {reg }}$ be its image in $\mathfrak{s}$. Then by the description of the fibers above we know that the restriction of $\mathfrak{A}$ to $\mathfrak{s}_{\text {reg }}$ is a torus bundle with typical fiber $A$. Its monodromy is $W$. More precisely:

$$
\left.\mathfrak{A}\right|_{\mathfrak{s}_{\mathrm{reg}}}=A \times{ }^{W} \mathfrak{a}_{\mathrm{reg}}^{*} \text {. }
$$

Local structure: The isomorphism (5.11) can be generalized to describe the local structure of $\mathfrak{A}$ in the neighborhood of any point $s \in \mathfrak{s}$. Choose $a \in \mathfrak{a}^{*}$ lying over $s$ and let

$$
\mathfrak{a}_{a}^{*}:=\left\{b \in \mathfrak{a}^{*} \mid\left\langle b \mid \alpha^{\vee}\right\rangle \neq 0 \text { for all } \alpha \in \Delta \backslash \Delta_{a}\right\} .
$$

This is also the set of all $b \in \mathfrak{a}^{*}$ with $\Delta_{b} \subseteq \Delta_{a}$ and also the complement of all reflection hyperplanes which do not contain $a$. The isotropy group $W_{a}$ is the Weyl group of $\Phi_{a}$. Moreover, the morphism $\mathfrak{s}_{a}:=\mathfrak{a}_{a}^{*} / W_{a} \rightarrow \mathfrak{s}$ is étale. Then

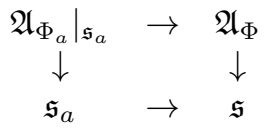

is a pull-back diagram. In other words, $\mathfrak{A}_{\Phi_{a}}$ and $\mathfrak{A}_{\Phi}$ are isomorphic over $\mathfrak{s}_{a}$. Proof: Let $\tilde{\mathfrak{a}}_{a}^{*}:=\mathfrak{s}_{a} \times_{\mathfrak{s}} \mathfrak{a}^{*}$. Then $\mathfrak{a}_{a}^{*}$ is a connected component of $\tilde{\mathfrak{a}}_{a}^{*}$. Its stabilizer in $W$ is $W(a)$ and all other components are $W$-translates. Now let $Y$ be any $S_{a}$-scheme. One checks

$$
\begin{aligned}
\operatorname{Mor}^{\Phi}\left(Y \times_{\mathfrak{s}} \mathfrak{a}^{*}, A\right) & =\operatorname{Mor}^{\Phi}\left(Y \times_{\mathfrak{s}_{a}} \tilde{\mathfrak{a}}_{a}^{*}, A\right) \\
& =\operatorname{Mor}^{\Phi_{a}}\left(Y \times_{\mathfrak{s}_{a}} \mathfrak{a}_{a}^{*}, A\right)=\operatorname{Mor}^{\Phi_{a}}\left(Y \times_{\mathfrak{s}} \mathfrak{a}^{*}, A\right) .
\end{aligned}
$$

Thus, the universal property implies $\mathfrak{A}_{\Phi} \times_{\mathfrak{s}} \mathfrak{s}_{a}=\left.\mathfrak{A}_{\Phi_{a}}\right|_{\mathfrak{s}_{a}}$.

Lie algebra: The Lie algebra of $\mathfrak{A}$ is a vector bundle over $\mathfrak{s}$. Taking for $Y$ the spectrum of $\mathbb{C}[X] /\left(X^{2}\right)$ one obtains

$$
\text { Lie } \mathfrak{A}_{\Phi}=T_{\mathfrak{s}}^{*},
$$

where $T_{\mathfrak{s}}^{*}$ is the cotangent bundle of $\mathfrak{s}$.

Symplectic structure: The isomorphism (5.15) implies that the tangent space of $\mathfrak{A}$ in a point of the identity section is of the form $T \oplus T^{*}$. Thus it carries a symplectic structure. We claim that in fact $\mathfrak{A}$ has a global symplectic structure; i.e., there is a natural closed and non-degenerate 2 -form $\omega$ on $\mathfrak{A}$. It is constructed as follows. First, $A \times \mathfrak{a}^{*}$ is the cotangent bundle of $A$, hence symplectic. The form is invariant under the natural $W$-action. Thus it pushes down to a symplectic structure on $A \times{ }^{W} \mathfrak{a}_{\mathrm{reg}}^{*}$. By (5.11), this space is isomorphic to the open subset $\mathfrak{A}_{\text {reg }}:=\left.\mathfrak{A}\right|_{\mathfrak{s}_{\text {reg }}}$ of $\mathfrak{A}$. We show that the symplectic form on $\mathfrak{A}_{\text {reg }}$ extends to a non-degenerate symplectic form on $\mathfrak{A}$.

Since $\mathfrak{A}$ is affine, we have to check this only in codimension one. The complement of $\mathfrak{A}_{\text {reg }}$ in $\mathfrak{A}$ is the part of $\mathfrak{A}$ sitting over the ramification divisor in $\mathfrak{s}$. The components of this divisor correspond to the conjugacy classes of roots. Choosing one component and one root belonging to this component and using (5.13) we are reduced to the case where the root system is of type $A_{1}$. Using (5.8), we may additionally assume that $\mathfrak{A}$ splits as the product of a torus and 
$\mathfrak{A}_{\Phi^{\prime}}$, where $\Phi^{\prime}$ is of rank one. Thus, we may assume that $\Phi=\Phi^{\prime}$ is of rank one.

Then we are in the case of the example in the beginning of this section. Recall that the coordinate ring of $\mathfrak{A}$ was generated by $a, b$, and $s$ with the relation $a^{2}-s b^{2}=1$. Moreover $s=t^{2}$, where $t$ is the coordinate of $\mathfrak{a}^{*}=\mathbb{C}$. Let $x$ be the coordinate of $A \cong \mathbb{C}^{\times}$. Then $x=a+t b$ is an eigenvalue of the matrix (5.1); i.e., the map

$$
\left(\begin{array}{cc}
a & s b \\
b & a
\end{array}\right) \mapsto(a+t b, t) \in \mathbb{C}^{\times} \times \mathbb{C}
$$

$$
\omega=\frac{d x}{x} \wedge d t=(a-t b)(d a+b d t+t d b) \wedge d t=(a d a-s b d b) d t+(a d b-b d a) \wedge t d t .
$$

From $a^{2}-s b^{2}=1$ it follows that the first summand vanishes. Thus

$$
\omega=\frac{1}{2}(a d b-b d a) \wedge d s
$$

is regular on all of $\mathfrak{A}$. More precisely, $\omega$ is the restriction of a 2 -form $\tilde{\omega}$ on $\mathbb{C}^{3}$ to $\mathfrak{A}$. Since $\mathfrak{A}$ is the zero-set of $f:=a^{2}-s b^{2}-1$ and because of

$$
\tilde{\omega} \wedge d f=(f+1) d a \wedge d b \wedge d s,
$$

the restriction $\omega$ is non-degenerate.

\section{The EXTENSION PROPERTY}

In this section, we prove a crucial property of the group scheme $\mathfrak{A}_{\Phi}$. It says that certain rational sections extend automatically to regular sections. This phenomenon is related to the group schemes introduced by Bruhat-Tits in [BrTi], 4.4. The difference is that there the base scheme is assumed to be just one-dimensional.

6.1. Definition. Let $S$ be an irreducible scheme with generic point $\eta$ and let $\mathfrak{G} / S$ be a group scheme acting on an $S$-scheme $X / S$. Let $X_{\eta}$ be the fiber of $X$ over $\eta$. The action of $\mathfrak{G}$ on $X$ is called saturated if for any section $u_{\eta}: \eta \rightarrow \mathfrak{G}$ of $\mathfrak{G}$ over $\eta$, the following are equivalent:

i) $u_{\eta}$ extends to a section of $\mathfrak{G}$ over $S$.

ii) The automorphism $\varphi_{\eta}$ of $X_{\eta}$ induced by $u_{\eta}$ extends to an $S$-automorphism $\varphi$ of $X$.

Clearly, only the implication $\overrightarrow{i i}) \Rightarrow$ i) is a problem. To formulate the results we need a restriction:

6.2. Definition. Let $S$ be an irreducible normal affine scheme with generic point $\eta$. An $S$-scheme $X$ is good if it is Noetherian, affine, faithfully flat over $S$ and if the generic fiber $X_{\eta}$ is geometrically irreducible.

Now we show that torus actions on good schemes are saturated. More precisely:

6.3. Lemma. Let $S$ be an irreducible normal $\mathbb{C}$-scheme and $X$ a good $S$-scheme. Let $\mathfrak{T} / S$ be a torus acting on $X / S$. Let $\eta$ be the generic point of $S$ and assume that the action of $\mathfrak{T}_{\eta}$ on $X_{\eta}$ has a finite kernel. Then the action of $\mathfrak{T} / S$ on $X / S$ is saturated. 
Proof. The torus $\mathfrak{T}$ splits over a Galois cover $\tilde{S} \rightarrow S$. It is easy to see that it suffices to show the assertion for $\tilde{\mathfrak{T}}=\mathfrak{T} \times{ }_{S} \tilde{S}$ acting on $\widetilde{X}=X \times_{S} \tilde{S}$. Thus, we may assume from the outset that $\mathfrak{T}=T \times_{\mathbb{C}} S$ is split, where $T$ is a torus over $\mathbb{C}$.

The normality of $S$ implies that a section $u_{\eta}$ extends to $S$ if and only if it extends in codimension one. Thus, after changing the base scheme $S$, we may assume that $S=\operatorname{Spec} V$, where $V$ is a discrete valuation ring. Let $t \in V$ be a uniformizer. Then $X=\operatorname{Spec} R$ is an affine scheme, where $R$ is a faithfully flat $V$-algebra. This means in particular that $t$ is not a zero-divisor in $R$; i.e., the homomorphism $R \rightarrow R\left[t^{-1}\right]$ is injective. Moreover, $X_{\eta}$ being irreducible means that $R\left[t^{-1}\right]$ is a domain. Hence, $R$ is a domain, as well.

Choose an isomorphism $T \cong\left(\mathbb{C}^{\times}\right)^{r}$. Then we can write

$$
u_{\eta}=\left(t^{d_{1}}, \ldots, t^{d_{r}}\right)\left(v_{1}, \ldots, v_{r}\right)=\lambda(t) \cdot v,
$$

where $d_{\nu} \in \mathbb{Z}$ and where $v_{i} \in V$ is invertible. Since $v$ is regular over $S$, we may replace $u_{\eta}$ by $\lambda$. Moreover, since $u_{\eta}=\lambda$ takes values in a one-dimensional subtorus we may assume $T=\mathbb{C}^{\times}$and $u_{\eta}=t^{d}$ with $d \in \mathbb{Z}$. All we have to show now is $d=0$.

Suppose $d \neq 0$. The action of $T$ on $X$ corresponds to a $\mathbb{Z}$-grading $R=\bigoplus_{m} R_{m}$. Let $\varphi$ be the automorphism of Definition 6.1[ii). Then $\varphi(f)=t^{d m} f$ for all $f \in R_{m}$. Since $\varphi$ is invertible we see that multiplication by $t$ is bijective on $R_{m}$ for all $m \neq 0$. Put $I:=t R$ and $J:=\bigcap_{n>0} I^{n}$. Because $R$ is a domain either $J=0$ or $I=R$ (Krull, see e.g. AtMa, 10.18). Suppose $J=0$. Since $R_{m} \subseteq J$ for $m \neq 0$ this would mean that $A$ is acting trivially on $X$, contradicting the assumption that the kernel of the action is finite. Thus $I=R$. But then $t$ is invertible in $R$, contradicting the assumption that $X$ is faithfully flat over $S$.

In the next lemma we show that the actions of $\mathfrak{A}_{\Phi}$ are almost saturated, even in a formal sense. For this, we use two ideas: 1 . the saturatedness of tori, and 2. the following simple fact: let $f(x)$ be a rational function with $f(-x)=f(x)^{-1}$. Then $f$ is defined and non-zero in $x=0$.

6.4. Lemma. Let $X$ be a good scheme over $\mathfrak{s}=\mathfrak{a}^{*} / W$. Assume that $\mathfrak{A}_{\Phi}$ is acting on $X$ such that the action over the generic point of $\mathfrak{s}$ is faithful. Let $\widehat{\mathfrak{s}}$ be the formal neighborhood of 0 in $\mathfrak{s}$ and let $\eta$ be its generic point. Let $u$ be a section of $\mathfrak{A}_{\Phi}$ over $\eta$ such that the action of $u$ on $X \times_{\mathfrak{s}} \eta$ extends to an automorphism of $X \times_{\mathfrak{s}} \widehat{\mathfrak{s}}$. Then $u$ extends to a section of $\mathfrak{A}_{W}$ over $\widehat{\mathfrak{s}}$.

Proof. First observe that $\mathfrak{A}_{\Phi}$ is an open subset of $\mathfrak{A}_{W}$ and that both coincide over $\mathfrak{s}_{\text {reg. }}$. We have to show that the morphism $u: \eta \rightarrow \mathfrak{A}_{\Phi}$ extends to a morphism $\widehat{\mathfrak{s}} \rightarrow \mathfrak{A}_{W}$. Again, since $\widehat{\mathfrak{s}}$ is normal, it suffices to check this in codimension one.

Let $\widehat{\mathfrak{s}}_{\text {reg }}=\widehat{\mathfrak{s}} \cap \mathfrak{s}_{\text {reg }}$ be the complement of the ramification divisor $D$. Since $\mathfrak{A}_{\Phi}$ is a torus over $\widehat{\mathfrak{\mathfrak { s }}}_{\text {reg }}$ we can invoke Lemma 6.3 and conclude that $u$ is regular over $\widehat{\mathfrak{s}}_{\text {reg }}$.

It remains to study regularity in $D$. For a root $\alpha \in \Delta$, let $D_{\alpha}$ be the image of the reflection hyperplane $H_{\alpha}$ in $\mathfrak{s}$. Then the $D_{\alpha}$ are precisely the irreducible components of $D$ with $D_{\alpha}=D_{\beta}$ if and only if $W \alpha=W \beta$.

Let $\widehat{\mathfrak{a}}^{*}$ be the formal neighborhood of $\mathfrak{a}^{*}$ in 0 . Moreover, let $V$ be the local ring of $\widehat{\mathfrak{a}}^{*}$ in $\widehat{\mathfrak{a}}^{*} \cap H_{\alpha}$ and let $\widehat{\mathfrak{a}}_{\alpha}^{*}$ be its spectrum. Then $V$ is a discrete valuation ring with valuation denoted by $v_{\alpha}$ and uniformizer $\alpha^{\vee}$.

Put $W_{\alpha}:=\left\{1, s_{\alpha}\right\}$ and $\widehat{\mathfrak{s}}_{\alpha}:=\widehat{\mathfrak{a}}_{\alpha}^{*} / W_{\alpha}$. Then there is a flat morphism $\widehat{\mathfrak{s}}_{\alpha} \rightarrow \widehat{\mathfrak{s}}$ with the closed point of $\widehat{\mathfrak{s}}_{\alpha}$ mapping to the generic point of $D_{\alpha}$. Thus we have to show that the rational section $u$ is regular over $\widehat{\mathfrak{s}}_{\alpha}$. 
Observe that

$$
\widehat{\mathfrak{s}}_{\alpha} \times \mathfrak{s} \mathfrak{a}^{*}=W \times{ }^{W_{\alpha}} \widehat{\mathfrak{a}}_{\alpha}^{*} .
$$

The section $u$ corresponds therefore to a $W_{\alpha}$-equivariant rational morphism $\tilde{u}: \widehat{\mathfrak{a}}_{\alpha}^{*} \ldots \rightarrow \rightarrow A$. Now put $f:=\alpha \circ \tilde{u}$, which is a rational function on $\widehat{\mathfrak{a}}_{\alpha}^{*}$. The $W_{\alpha^{-}}$ equivariance of $\tilde{u}$ implies that

$$
f \circ s_{\alpha}=\alpha \circ \tilde{u} \circ s_{\alpha}=\alpha \circ s_{\alpha} \circ \tilde{u}=(\alpha \circ \tilde{u})^{-1}=f^{-1} .
$$

Because the valuation $v_{\alpha}$ is $s_{\alpha}$-invariant this implies that $v_{\alpha}(f)=0$; i.e., $f$ is actually defined and invertible on $\widehat{\mathfrak{a}}_{\alpha}^{*}$. Equation (6.3) implies moreover that $f^{2}-1$ vanishes on $H_{\alpha}$.

Now we consider the coroot $\alpha^{\vee}$ as a homomorphism $\mathbb{C}^{\times} \rightarrow A$ and define a morphism $b: \widehat{\mathfrak{a}}_{\alpha}^{*} \rightarrow A$ by $b=\alpha^{\vee}(f)$. Then the $W_{\alpha}$-equivariance of $\tilde{u}$ can be rewritten as

$$
\tilde{u} \circ s_{\alpha}=\tilde{u} \cdot b^{-1}
$$

Thus

$$
c:=\tilde{u}^{2} b^{-1}=\tilde{u} \cdot\left(\tilde{u} \circ s_{\alpha}\right)
$$

has values in $A^{W_{\alpha}}$. This group has at most two connected components, which implies that $c^{2}$ has values in the torus $A_{0}:=\left(A^{W_{\alpha}}\right)^{0}$. Now we use the fact that $u$ induces an action on all of $X \times_{\mathfrak{s}} \widehat{\mathfrak{s}}$. Thus also $c$ extends to an automorphism of $X \times_{\mathfrak{s}} \widehat{\mathfrak{s}}_{\alpha}$. Since it is part of a torus action, we conclude (Lemma 6.3) that $c$ is regular on $\widehat{\mathfrak{a}}_{\alpha}^{*}$. Thus, also $\tilde{u}^{4}$ and therefore $\tilde{u}$ are regular on $\widehat{\mathfrak{a}}_{\alpha}^{*}$.

Before we go on, we need to study the difference between sections of $\mathfrak{A}_{W}$ and sections of $\mathfrak{A}_{\Phi}$. Recall that each element $t \in A^{W}$ gives rise to a constant section, also denoted by $t$, of $\mathfrak{A}_{W}$.

6.5. Lemma. Let $\widehat{\mathfrak{s}}$ be the formal neighborhood of a point $s \in \mathfrak{s}$. Then

$$
\Gamma\left(\widehat{\mathfrak{s}}, \mathfrak{A}_{W}\right)=A^{W} \cdot \Gamma\left(\widehat{\mathfrak{s}}, \mathfrak{A}_{\Phi}\right) .
$$

Proof. For an algebraic group $E$, let $\pi_{0}(E)=E / E^{0}$ be its group of components. It suffices to show that

$$
A^{W} \rightarrow \pi_{0}\left(\mathfrak{A}_{W, s}\right)
$$

is surjective. Indeed, let $u$ be a section of $\mathfrak{A}_{W}$ over $\widehat{\mathfrak{s}}$. Let $u(s) \in \mathfrak{A}_{W, s}$ be its value at $s$. Then there is $t_{0} \in A^{W}$ with $u(s) \cdot t_{0}^{-1} \in \mathfrak{A}_{W, s}^{0} \subseteq \mathfrak{A}_{\Phi, s}$. But then $u_{1}:=t_{0}^{-1} \cdot u$ is a section of $\mathfrak{A}_{\Phi}$.

To show surjectivity of (6.7), let $a \in \mathfrak{a}^{*}$ be a lift of $s$. Because of $\pi_{0}\left(\mathfrak{A}_{W, s}\right)=$ $\pi_{0}\left(A^{W_{a}}\right)$ it suffices to show that $A^{W} \rightarrow \pi_{0}\left(A^{W_{a}}\right)$ is surjective or, equivalently, that the map between character groups is injective.

Let $\Delta_{\max } \subseteq \Lambda$ be the root system with Weyl group $W$ such that all coroots are primitive. Then $W_{a}$ corresponds to a subroot system $\Delta_{\max }^{\prime}$. Choose a system of simple roots $\Sigma \subseteq \Delta_{\max }$ such that $\Sigma^{\prime}=\Sigma \cap \Delta_{\max }^{\prime}$ is a set of simple roots for $\Delta_{\max }^{\prime}$. Then the map of character groups is

$$
\left(\Lambda /\left\langle\Delta_{\max }^{\prime}\right\rangle\right)_{\text {torsion }} \longrightarrow \Lambda /\left\langle\Delta_{\max }\right\rangle .
$$

The kernel is a torsion subgroup of the free group $\left\langle\Delta_{\max }\right\rangle /\left\langle\Delta_{\max }^{\prime}\right\rangle \cong \sum_{\alpha \in \Sigma \backslash \Sigma^{\prime}} \mathbb{Z} \alpha$, hence zero. 
Next, we investigate when there is actually a difference between $\mathfrak{A}_{W}$ and $\mathfrak{A}_{\Phi}$. Clearly this only happens if there is a root $\alpha$ with $\frac{1}{2} \alpha^{\vee} \in \Lambda^{\vee}$. These roots will be called special.

6.6. Lemma. Let $\alpha$ be a special root. Then $\Phi$ has a direct summand isomorphic to the root datum of $S O_{2 n+1}(\mathbb{C}), n \geq 1$, in which $\alpha$ is a short root.

Remark. The root datum of $S_{2 n+1}(\mathbb{C})$ is of course the root system $\mathrm{B}_{n}$, where $\Lambda$ is the root lattice. Observe that $B_{1}=A_{1}$ is included.

Proof. We may choose a set of simple roots $\Sigma \subseteq \Delta$ containing $\alpha$. Then $\langle\Lambda|$ $\left.\alpha^{\vee}\right\rangle=2 \mathbb{Z}$ implies $\left\langle\beta \mid \alpha^{\vee}\right\rangle \in\{0,-2\}$ for all $\beta \in \Sigma$. The classification of Dynkin diagrams shows that $\alpha$ is the short simple root of a subroot system of type $\mathrm{B}_{n}$. Let $\alpha_{1}, \ldots, \alpha_{n}$ be the short positive roots. By assumption, the corresponding halfcoroots $\frac{1}{2} \alpha_{1}^{\vee}, \ldots, \frac{1}{2} \alpha_{n}^{\vee}$ lie in $\Lambda^{\vee}$. Because of $\left\langle\alpha_{i} \mid \frac{1}{2} \alpha_{j}^{\vee}\right\rangle=\delta_{i j}$, we see that the lattice spanned by the $\alpha_{i}$ is a direct summand of $\Lambda$.

As already mentioned, any root $\alpha$ gives rise to a component $D_{\alpha}$ of the ramification divisor of $\mathfrak{a}^{*} \rightarrow \mathfrak{s}$. We call $D_{\alpha}$ special if $\alpha$ is special.

6.7. Lemma. Let $D \subseteq \mathfrak{s}$ be an irreducible divisor.

i) If $D$ is not a special divisor, then $\mathfrak{A}_{\Phi, s}=\mathfrak{A}_{W, s}$ for a generic point $s \in D$.

ii) If $D=D_{\alpha}$ is special, then $\mathfrak{A}_{\Phi, s}$ is of index 2 in $\mathfrak{A}_{W, s}$ for a generic point $s \in D$.

iii) If $D=D_{\alpha}$ is special, then there is an involution $e_{\alpha} \in A^{W}$ with $e_{\alpha}(s) \notin \mathfrak{A}_{\Phi, s}$ if and only if $s \in D$.

Proof. If $D$ is not part of the ramification divisor, then, for $s \in D$ generic, $\mathfrak{A}_{W, s}$ is a torus; hence $\mathfrak{A}_{\Phi, s}=\mathfrak{A}_{W, s}$. Otherwise, $D=D_{\alpha}$ for some root $\alpha$. If $\alpha$ is not special, then $\alpha \in \Delta_{\max }$. Thus $\mathfrak{A}_{\Phi, s}=\mathfrak{A}_{W, s} \cong \operatorname{ker} \alpha \times \mathbb{C}$ for $s \in D$ generic. Otherwise, $2 \alpha \in \Delta_{\max }$ and $\mathfrak{A}_{\Phi, s}$ is of index 2 in $\mathfrak{A}_{W, s}$. This shows $i$ ) and $i i$ ).

For iii) we may assume that $\alpha$ is a short root in a direct summand $\Phi^{\prime}$ of $\Phi$ which is of type $B_{n}$. Thus, there is a unique homomorphism $\varepsilon_{\alpha}: \Lambda \rightarrow\{ \pm 1\}$ with

$$
\varepsilon_{\alpha}(\chi)= \begin{cases}-1 & \text { if } \chi \text { is a short root of } \Phi^{\prime} \\ +1 & \text { if } \chi \text { is another root or if } \chi \text { is fixed by } W\end{cases}
$$

This homomorphism is clearly $W$-invariant and of order 2 . Thus, it can be interpreted as an involution in $e_{\alpha} \in A^{W}$. Let $s \in \mathfrak{s}$ and let $a \in \mathfrak{a}^{*}$ lie above $s$. Then $e_{\alpha}(s) \in \mathfrak{A}_{\Phi, s}$ if and only if $\varepsilon_{\alpha}\left(\Delta_{a}\right)=1$ if and only if $\Delta_{a}$ contains no short root of $\Phi^{\prime}$ if and only if $s \notin D_{\alpha}$.

Now we can give a criterion for an $\mathfrak{A}_{\Phi}$-action to be saturated:

6.8. Theorem. Assume $\mathfrak{A}_{\Phi} / \mathfrak{s}$ acts on a scheme $X / \mathfrak{s}$ which is good and assume that the action is generically effective. For $s \in \mathfrak{s}$, let $\widehat{\mathfrak{s}}_{s}$ be its formal neighborhood. Moreover, put $\widehat{X}_{s}:=X \times_{\mathfrak{s}} \widehat{\mathfrak{s}}_{s}$ and $\widehat{\mathfrak{A}}_{s}=\mathfrak{A}_{\Phi} \times_{\mathfrak{s}} \widehat{\mathfrak{s}}_{s}$. Then the following are equivalent:

i) For every $s \in \mathfrak{s}$, the action of $\widehat{\mathfrak{A}}_{s}$ on $\widehat{X}_{s}$ is saturated.

ii) The action of $\mathfrak{A}_{\Phi}$ on $X$ is saturated.

iii) Let $t \in A^{W}$ with $t \notin A^{\Phi}$. Then the rational action of $t$ on $X$ does not extend to an action on all of $X$. 
Proof. $i$ ) $\Rightarrow$ ii) Let $u$ be a rational section of $\mathfrak{A}_{\Phi}$ such that the action of $u$ on $X$ is regular. Then $u$ is regular over $\widehat{\mathfrak{s}}_{s}$ for every $s \in \mathfrak{s}$. Hence it is regular on all of $\mathfrak{s}$.

ii) $\Rightarrow$ iii) Let $t \in A^{W}$ whose rational action on $X$ is regular. Then $t$ is a regular section of $\mathfrak{A}_{\Phi}$, i.e., $t \in A^{\Phi}$.

iii) $\Rightarrow$ i) Let $u$ be a rational section of $\mathfrak{A}_{\Phi}$ over $\widehat{\mathfrak{s}}_{s}$ which induces an automorphism of $\widehat{X}_{s}$. Then Lemma 6.4 implies that $u$ is a regular section of $\mathfrak{A}_{W}$ over $\widehat{\mathfrak{s}}_{s}$, and we have to show that $u$ is actually a section of $\mathfrak{A}_{\Phi}$. Lemma 6.5 implies that there is $t_{0} \in A^{W}$ such that $u_{1}=u \cdot t_{0}^{-1}$ is regular over $\widehat{\mathfrak{s}}_{s}$. Thus, we may replace $u$ by $t_{0}$.

So assume $u \in A^{W}$. Now let $D \subset \mathfrak{s}$ be an irreducible divisor over which $u$ is not a section of $\mathfrak{A}_{\Phi}$. Then Lemma 6.7 tells us that $D=D_{\alpha}$ is a special divisor. If $s \notin D_{\alpha}$, then we may eliminate $D_{\alpha}$ by multiplying $u$ by the special involution $e_{\alpha}$ of Lemma 6.7 (ii). Thus, we may assume $s \in D_{\alpha}$. Then the action of $u$ on $X$ is regular outside the union of finitely many irreducible divisors which all pass through $s$. On the other side, the action of $u$ extends to an action on $\widehat{X}_{s}$. These two facts imply that the action of $u$ on $X$ is regular outside a subset of codimension $\geq 2$. Since $X$ is affine, the action is regular everywhere. Now iii) implies $u \in A^{\Phi}$. A fortiori, $u$ is a regular section of $\mathfrak{A}_{\Phi}$ over $\widehat{\mathfrak{s}}_{s}$.

Finally, we need that for given $W$ and $X$ at most one $\mathfrak{A}_{\Phi}$ can act in a saturated fashion.

6.9. Theorem. Let $\widehat{\mathfrak{s}}$ be the formal neighborhood of 0 in $\mathfrak{s}$ and let $\widehat{X} / \widehat{\mathfrak{s}}$ be good. Let $\Phi_{1}$ and $\Phi_{2}$ be two root systems with the same lattice and Weyl group. Assume that both $\mathfrak{A}_{\Phi_{1}} \times_{\mathfrak{s}} \widehat{\mathfrak{s}}$ and $\mathfrak{A}_{\Phi_{2}} \times_{\mathfrak{s}} \widehat{\mathfrak{s}}$ act on $X$ inducing the same action of their Lie algebra. Assume moreover that both group scheme actions are saturated. Then $\Phi_{1}=\Phi_{2}$.

Proof. Let $E \subseteq A^{W}$ be the group of constant sections which act on $X$. By saturatedness, we have $A^{\Phi_{1}}=E=A^{\Phi_{2}}$. This implies $\Phi_{1}=\Phi_{2}$.

\section{AUtOMORPHISMS OF SPHERICAL VARIETIES}

In this section, we apply the results of the preceding section to the cotangent bundle of spherical varieties. Our starting point is the following theorem.

7.1. Theorem. Let $X$ be a smooth affine spherical variety. Then there is a root system $\Phi_{X}$ with lattice $\Lambda_{X}$ (the group generated by the weight monoid) and Weyl group $W_{X}$ with:

i) The group scheme $\mathfrak{A}=\mathfrak{A}_{\Phi_{X}}$ acts on $Z:=T_{X}^{*}$ over $\mathfrak{s}:=\mathfrak{a}^{*} / W_{X}$.

ii) This action is uniquely determined by the requirement that the following diagram commutes:

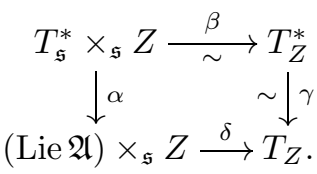

Here, $\alpha$ is induced by the isomorphism (5.15), $\beta$ is a pull-back of cotangent vectors via $Z \rightarrow \mathfrak{s}, \gamma$ comes from the symplectic structure on $Z$, and $\delta$ is the infinitesimal action of $\mathfrak{A}$ on $Z$.

iii) The action of $\mathfrak{A}$ on $Z$ is saturated.

Proof. See [Kn3, Thm. 4.1 and Thm. 7.8. 
It turns out that the data $\left(\Lambda_{X}, W_{X}\right)$ determine to a large extent the generic structure of $Z$. Let $L \subseteq G$ be the centralizer of $\mathfrak{a}^{*} \subseteq \mathfrak{t}^{*} \subseteq \mathfrak{g}^{*}$ under the coadjoint action. This is also the largest Levi subgroup of $G$ containing $T$ such that $\Lambda_{X} \subseteq$ $X(L)$. Let $L_{0}$ be the set of $g \in L$ with $\chi(l)=1$ for all $\chi \in \Lambda_{X}$. This is a normal subgroup of $L$ such that $A:=L / L_{0}$ is a torus with Lie algebra a.

7.2. Proposition. There is a dense open $W_{X}$-stable subset $\mathfrak{a}_{0}^{*} \subseteq \mathfrak{a}^{*}$ and an isomorphism

$$
\varepsilon: G / L_{0} \times \mathfrak{a}_{0}^{*} \stackrel{\sim}{\longrightarrow} Z \times{ }_{\mathfrak{s}} \mathfrak{a}_{0}^{*}
$$

with:

i) $\varepsilon$ is G-equivariant.

ii) $\varepsilon$ is compatible with the moment map, i.e., the diagram

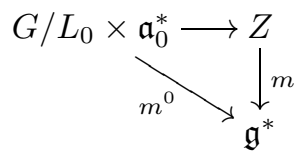

commutes, where $m^{0}$ is the morphism $\left(g L_{0}, \xi\right) \mapsto g \xi$.

iii) $\varepsilon$ is compatible with the invariant moment map, i.e., the diagram

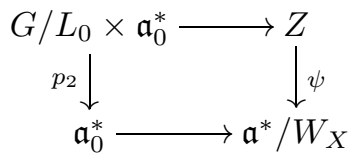

commutes, where $p_{2}$ is the projection to the second factor.

iv) Let $W_{X}$ act on $Z \times_{\mathfrak{s}} \mathfrak{a}_{0}^{*}$ by acting on the second factor. Then $\varepsilon$ is $W_{X}$ equivariant, where $w \in W_{X}$ acts on $G / L_{0} \times \mathfrak{a}_{0}^{*}$ according to the formula

$$
{ }^{w}\left(g L_{0}, \xi\right)=\left(g t_{\bar{w}}(\xi) \bar{w}^{-1} L_{0}, w \xi\right) .
$$

Here, $\bar{w}$ is a lift of $w$ to $N_{G}\left(\mathfrak{a}_{X}^{*}\right)$ and $t_{\bar{w}}: \mathfrak{a}_{0}^{*} \rightarrow A$ is some morphism.

v) $\varepsilon$ is compatible with the $\mathfrak{A}_{X}$-action: because of $\mathfrak{A}_{X} \times_{\mathfrak{s}} \mathfrak{a}_{0}^{*}=A \times \mathfrak{a}_{0}^{*}$, this is just an $A$-action which coincides with the right $A$-action on $G / L_{0}$.

Proof. Most assertions follow from $\mathrm{Kn} 2$, especially $\S 4$. The main point is that the closure of $m(Z)$ coincides with the closure of $G \mathfrak{a}^{*}$ inside $\mathfrak{g}^{*}$ ( $\underline{\mathrm{Kn} 2}$, 3.3). Thus $\psi: G \times{ }^{L} m^{-1}\left(\mathfrak{a}^{*}\right) \rightarrow Z$ is dominant and even generically finite but $\psi$ is in general not birational and $m^{-1}\left(\mathfrak{a}^{*}\right)$ is not irreducible. Let $B$ be the Borel subgroup of $G$ and $X_{0}=B x_{0}$ be the open $B$ orbit in $X$. Then we replace $\mathfrak{a}^{*}$ by an open subset $\mathfrak{a}_{0}^{*}$ (making $\psi$ finite) and $m^{-1}\left(\mathfrak{a}_{0}^{*}\right)$ by its intersection $\Sigma$ with $T_{X_{0}}^{*}$. Moreover, we replace the target $Z$ by $\tilde{Z}:=Z \times{ }_{\mathfrak{t}^{*} / W} \mathfrak{a}_{0}^{*}$. Then $\Sigma$ is irreducible and $\widehat{\psi}: G \times^{L} \Sigma \rightarrow \tilde{Z}$ is an open embedding. The image is an irreducible component $\widehat{T}_{X}=T_{X}^{*} \times_{\mathfrak{a}^{*} / W_{X}} \mathfrak{a}_{0}^{*}$ of $\tilde{Z}$. Since $L_{0}$, the principal isotropy group, acts trivially on $\Sigma$, the action factors through $A=L / L_{0}$. Moreover, after possibly shrinking $\mathfrak{a}_{0}^{*}$, the action of $A$ on $\Sigma$ is free and $\Sigma \rightarrow \mathfrak{a}_{0}^{*}$ is a quotient. Thus, again after shrinking $\mathfrak{a}_{0}^{*}$, there is an isomorphism $\Sigma \cong A \times \mathfrak{a}_{0}^{*}$. From this we get a map

$$
\varepsilon: G / L_{0} \times \mathfrak{a}_{0}^{*}=G \times{ }^{L} \Sigma \hookrightarrow \widehat{T}_{X} \rightarrow T_{X}^{*}
$$

Now all assertions are clear by construction except for possibly $i v$ ) 
The $W_{X}$-action is of course the one on $\widehat{T}_{X}$. Thus, the $W_{X}$-action commutes with the $G$-action and the second projection to $\mathfrak{a}_{0}^{*}$ is $W_{X}$-equivariant. Therefore, the action of $w \in W_{X}$ must have the form

$$
{ }^{w}\left(g L_{0}, \xi\right)=\left(g n_{w}(\xi) L_{0}, w \xi\right)
$$

where $n_{w}$ is a morphism $\mathfrak{a}_{0}^{*} \rightarrow N_{G}\left(L_{0}\right) / L_{0}$. Now, the fact that $m$ is $W_{X}$-invariant implies that $n_{w}(\xi) w \xi=\xi$ for all $\xi \in \mathfrak{a}_{0}^{*}$. Thus, $t_{\bar{w}}(\xi):=n_{w}(\xi) \bar{w} \in L / L_{0}=A$, as claimed.

Later on, we also need the following complements to Theorem 7.1 .

7.3. Corollary. With the notation above:

i) The moment map is $\mathfrak{A}$-invariant, i.e., $m(a z)=m(z)$ for all $(a, z) \in \mathfrak{A} \times{ }_{\mathfrak{s}} Z$.

ii) The $\mathfrak{A}$-action is compatible with the symplectic structure of $Z$; i.e., the following formula holds:

$$
\mu^{*} \omega_{Z}=p r_{1}^{*} \omega_{\mathfrak{A}}+p r_{2}^{*} \omega_{Z}
$$

Here $p_{1}: \mathfrak{A} \times_{\mathfrak{s}} Z \rightarrow \mathfrak{A}$ is the first projection and $\mu, p r_{2}: \mathfrak{A} \times_{\mathfrak{s}} Z \rightarrow Z$ the multiplication and the second projection, respectively. Moreover, $\omega_{\mathfrak{A}}$ and $\omega_{Z}$ are the symplectic forms on $\mathfrak{A}$ and $Z$, respectively.

Proof. i) follows from $i i$ ) and $v$ ) above.

ii) As in the proof above we write $G / L_{0} \times \mathfrak{a}_{0}^{*}=G \times{ }^{L} \Sigma$ with $\Sigma \cong A \times \mathfrak{a}_{0}^{*}$. Then $\Sigma$ is a Hamiltonian $A$-variety with the second projection as moment map. Now choose coordinates $t_{\nu}$ of $A$ and compatible coordinates $x_{\nu}$ of $\mathfrak{a}_{0}^{*}$. Then the symplectic form on $\Sigma$ has the form

$$
\omega_{\Sigma}=\sum_{\nu} \frac{d t_{\nu}}{t_{\nu}} \wedge d x_{\nu}+\omega_{0},
$$

where $\omega_{0}$ is a 2 -form involving only the coordinates $x_{\nu}$. The pull-back $\tilde{\mathfrak{A}}$ of $\mathfrak{A}_{X}$ to $\mathfrak{a}_{0}^{*}$ is the trivial group scheme with fiber $A$. Thus we have

$$
\tilde{\mathfrak{A}} \times \mathfrak{a}_{0}^{*} \Sigma=A \times A \times \mathfrak{a}_{0}^{*} .
$$

Denote the coordinates of the three factors by $s_{\nu}, t_{\nu}$, and $x_{\nu}$, respectively. Then $p r_{1}, p r_{2}$, and $\mu$ are, respectively,

$$
(s, t, x) \mapsto(s, x),(t, x),(s t, x) .
$$

A very short calculation shows that (7.8) holds for the $\tilde{\mathfrak{A}}$-action on $\Sigma$. Thus, it also holds for the $\tilde{\mathfrak{A}}$-action on $G \times{ }^{L} \Sigma$ by $G$-equivariance and therefore for the $\mathfrak{A}_{X}$-action on $Z$.

Now we are able to determine the automorphisms of formal neighborhoods of closed orbits in $T_{X}^{*}$.

7.4. Theorem. Let $X$ be a smooth affine spherical variety and $Z=T_{X}^{*}$. Let $\widehat{\mathfrak{s}}$ be the formal neighborhood of a point $s$ in $\mathfrak{s}$. Put $\widehat{Z}:=Z \times_{\mathfrak{s}} \widehat{\mathfrak{s}}$ and let $\varphi$ be a $G$-equivariant automorphism of $\widehat{Z}$ such that both the moment map $\widehat{Z} \rightarrow \mathfrak{g}^{*}$ and the invariant moment map $\widehat{Z} \rightarrow \widehat{\mathfrak{s}}$ are $\varphi$-invariant. Then $\varphi$ is induced by a (unique) section $u$ of $\mathfrak{A}_{X}$ over $\widehat{\mathfrak{s}}$. 
Proof. Let $\dot{\mathfrak{s}}$ be the generic point of $\widehat{\mathfrak{s}}$ and $\dot{Z}:=\widehat{Z} \otimes_{\mathfrak{s}} \dot{\mathfrak{s}}=Z \times_{\mathfrak{s}} \dot{\mathfrak{s}}$. We claim that it suffices to construct a section $\dot{u}$ over $\dot{\mathfrak{s}}$. In fact, the morphism $Z \rightarrow \mathfrak{s}$ satisfies the conditions of Theorem 6.8 (the generic fiber is irreducible by construction; see Kn1, 6.6 for the faithful flatness). Thus, since the action of $\mathfrak{A}_{X}$ on $Z$ is saturated (Theorem 7.1) the same holds for the action on $\widehat{Z}$ (Theorem 6.8). Thus, $\dot{u}$ extends to a section $u$ over $\widehat{\mathfrak{s}}$. The automorphism induced by $u$ coincides with $\varphi$ over $\dot{\mathfrak{s}}$. So it coincides over all of $\widehat{\mathfrak{s}}$ since $\widehat{Z} \rightarrow \widehat{\mathfrak{s}}$ is faithfully flat.

To construct $\dot{u}$, let $\dot{\mathfrak{a}}^{*}=\dot{\mathfrak{s}} \times \mathfrak{s} \mathfrak{a}^{*}$. Thus, via the morphism $\dot{\mathfrak{a}}^{*} \rightarrow \mathfrak{a}_{0}^{*}$, the isomorphism $\varepsilon$ in Proposition 7.2 yields an isomorphism

$$
G / L_{0} \times \dot{\mathfrak{a}}^{*} \stackrel{\sim}{\longrightarrow} \dot{Z} \times \dot{\mathfrak{s}}^{*} .
$$

Since $\dot{Z} \rightarrow \dot{\mathfrak{s}}$ is $\varphi$-invariant, $\varphi$ extends to an automorphism $\tilde{\varphi}$ of the right-hand side and therefore of $G / L_{0} \times \dot{\mathfrak{a}}^{*}$. Because $\tilde{\varphi}$ is $G$-equivariant and because the projection to the second factor is $\tilde{\varphi}$-invariant, it follows that there is a morphism $t: \dot{\mathfrak{a}}^{*} \rightarrow \operatorname{Aut}^{G}\left(G / L_{0}\right)=N_{G}\left(L_{0}\right) / L_{0}$ such that

$$
\tilde{\varphi}\left(g L_{0}, \xi\right)=\left(g t(\xi) L_{0}, \xi\right) .
$$

Now the invariance of the moment map $\dot{Z} \rightarrow \mathfrak{g}^{*}$ implies that $t(\xi) \xi=\xi$. Thus $t$ takes values in $C_{G}\left(\mathfrak{a}^{*}\right) / L_{0}=L / L_{0}=A$. Finally, we use that $\tilde{\varphi}$ commutes with the $W_{X}$ action. From

$$
\begin{aligned}
& w \tilde{\varphi}\left(e L_{0}, \xi\right)=w\left(t(\xi) L_{0}, \xi\right)=\left(t(\xi) \bar{w}^{-1} t_{\bar{w}}(\xi), w \xi\right), \\
& \tilde{\varphi} w\left(e L_{0}, \xi\right)=\tilde{\varphi}\left(\bar{w}^{-1} t_{\bar{w}}(\xi) L_{0}, w \xi\right)=\left(\bar{w}^{-1} t_{\bar{w}}(\xi) t(w \xi) L_{0}, w \xi\right),
\end{aligned}
$$

we obtain $t(w \xi)=w t(\xi) w^{-1}$; i.e., $t: \dot{\mathfrak{a}}^{*} \rightarrow A$ is $W_{X}$-equivariant. Thus $t$ descends to a section $\dot{u}$ of $\mathfrak{A}_{X}$ over $\dot{\mathfrak{s}}$ inducing $\varphi$.

\section{Comparison Results}

In this section, we provide some tools to pass from the algebraic to the smooth category.

Let $K$ be a connected compact Lie group. If $V$ is a, possibly infinite-dimensional, topological representation of $K$, let

$$
V^{\text {fin }}:=\{v \in V \mid \operatorname{dim}\langle K v\rangle<\infty\}
$$

be its subrepresentation of $K$-finite vectors. It is a consequence of the Peter-Weyl theorem that $V^{\text {fin }}$ is dense in $V$ whenever $V$ is locally convex. For the next lemma observe that every $K$-equivariant vector bundle $\mathcal{E}$ on a homogeneous space has a real algebraic structure. Let $\Gamma^{\text {alg }}$ (resp. $\Gamma^{\infty}$ ) denote algebraic (resp. smooth) sections.

8.1. Lemma. Let $\mathcal{E} \rightarrow K / H_{\mathbb{R}}$ be a $K$-equivariant vector bundle of finite rank. Then there is an isomorphism

$$
\Gamma^{\mathrm{alg}}\left(K / H_{\mathbb{R}}, \mathcal{E}\right) \stackrel{\sim}{\rightarrow} \Gamma^{\infty}\left(K / H_{\mathbb{R}}, \mathcal{E}\right)^{\mathrm{fin}} .
$$

Proof. Let $E$ be the fiber of $\mathcal{E}$ over the base point $e H_{\mathbb{R}} \in K / H_{\mathbb{R}}$. This is a finitedimensional representation of $H_{\mathbb{R}}$, and we have $\mathcal{E}=K \times{ }^{H_{\mathbb{R}}} E$. Then,

$$
\Gamma\left(K / H_{\mathbb{R}}, \mathcal{E}\right)=\operatorname{Mor}^{H_{\mathbb{R}}}(K, E)
$$

both in the algebraic and in the smooth category. Let $U$ be any irreducible representation of $K$. Then, by Frobenius reciprocity, a $K$-homomorphism $U \rightarrow \Gamma\left(K / H_{\mathbb{R}}, \mathcal{E}\right)$ 
is the "same" as an $H_{\mathbb{R}}$-homomorphism $\operatorname{res}_{H_{\mathbb{R}}} U \rightarrow E$. The latter is independent of the category. Thus we have

$$
\operatorname{Hom}^{K}\left(U, \Gamma^{\operatorname{alg}}\left(K / H_{\mathbb{R}}, \mathcal{E}\right)\right) \stackrel{\sim}{\rightarrow} \operatorname{Hom}^{K}\left(U, \Gamma^{\infty}\left(K / H_{\mathbb{R}}, \mathcal{E}\right)\right),
$$

implying (8.3).

Here is now the comparison statement:

8.2. Lemma. Let $Y=K x$ be a $K$-orbit in a smooth real-algebraic $K$-manifold M. Let $\mathcal{I}_{Y} \subseteq \mathcal{C}^{\infty}(\mathrm{M})$ be the ideal of smooth functions vanishing in $Y$ and let $\mathcal{C}_{Y}^{\infty}(\mathrm{M})$ be the $\mathcal{I}_{Y}$-adic completion of $\mathcal{C}^{\infty}(\mathrm{M})$ (i.e., the ring of formal power series along $Y)$. On the other hand, let $\mathbb{R}[\mathrm{M}]$ be the ring of regular functions on $\mathrm{M}$, let $I_{Y}=\mathcal{I}_{Y} \cap \mathbb{R}[\mathrm{M}]$ be the ideal of regular functions vanishing in $Y$ and let $\mathbb{R} \llbracket \mathrm{M} \rrbracket$ be the $I_{Y}$-adic completion of $\mathbb{R}[\mathrm{M}]$. Then there are natural isomorphisms

$$
\mathcal{C}_{Y}^{\infty}(\mathrm{M})^{\mathrm{fin}} \cong \mathbb{R} \llbracket \mathrm{M} \rrbracket^{\text {fin }} \cong \mathbb{R}[\mathrm{M}] \otimes_{\mathbb{R}[\mathrm{M}]^{K}} \mathbb{R} \llbracket \mathrm{M} \rrbracket^{K} .
$$

Proof. The second isomorphism is a theorem of Magid Mag.

For the first isomorphism, put $H_{\mathbb{R}}=K_{x}$ and choose an $H_{\mathbb{R}}$-stable complement $N$ to $\mathfrak{k} x$ in $T_{\mathrm{M}, x}$. Then Luna's slice theorem asserts that, in the étale topology, a neighborhood of $Y$ in $\mathrm{M}$ is isomorphic to a neighborhood of $K / H_{\mathbb{R}}$ in the fiber bundle $K \times{ }^{H_{\mathbb{R}}} N$. A fortiori, this holds in the Hausdorff topology, too. Thus, we may replace $\mathrm{M}$ by $K \times{ }^{H_{\mathbb{R}}} N$.

Let $E_{n}:=\bigoplus_{i=0}^{n-1} S^{i} N^{*}$ and $\mathcal{E}_{n}:=K \times{ }^{H_{\mathbb{R}}} E_{n}$. Then $\mathbb{R}[\mathrm{M}] / I_{Y}^{n}$ and $\mathcal{C}^{\infty}(\mathrm{M}) / \mathcal{I}_{Y}^{n}$ are the algebraic, resp. smooth, sections of the vector bundle $\mathcal{E}_{n}$. Thus Lemma 8.1 implies that

$$
\mathbb{R}[\mathrm{M}] / I_{Y}^{n} \cong\left(\mathcal{C}^{\infty}(\mathrm{M}) / \mathcal{I}_{Y}^{n}\right)^{\text {fin }}
$$

Now observe that, for any sequence $V_{n}$ of $K$-representations, the following holds:

$$
\left(\prod_{n} V_{n}^{\mathrm{fin}}\right)^{\mathrm{fin}}=\left(\prod_{n} V_{n}\right)^{\mathrm{fin}} .
$$

This in turn implies that

and therefore

$$
\left(\lim _{\longleftarrow} V_{n}^{\text {fin }}\right)^{\text {fin }}=\left(\lim _{\longleftarrow} V_{n}\right)^{\text {fin }}
$$

$$
\left(\varliminf_{\lim } \mathbb{R}[\mathrm{M}] / I_{Y}^{n}\right)^{\mathrm{fin}}=\left(\varliminf_{\longleftarrow}^{\lim }\left(\mathcal{C}^{\infty}(\mathrm{M}) / \mathcal{I}_{Y}^{n}\right)^{\mathrm{fin}}\right)^{\mathrm{fin}}=\left(\varliminf_{\longleftarrow} \mathcal{C}^{\infty}(\mathrm{M}) / \mathcal{I}_{Y}^{n}\right)^{\mathrm{fin}} .
$$

Our second comparison statement is concerned with lifting of smooth maps. More precisely, consider the following diagram in the category of manifolds:

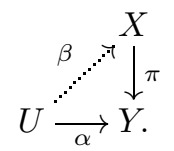

Given $\alpha$ and $\pi$, we say that $\alpha$ lifts to $X$ if there is $\beta$ with $\alpha=\pi \circ \beta$. For every $u \in U, x \in X$ with $y:=\alpha(u)=\pi(y)$ we obtain homomorphisms $\widehat{\alpha}$ and $\widehat{\pi}$ between completions:

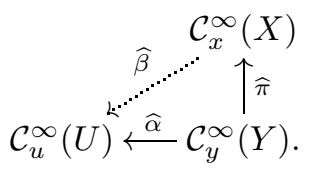


We say that $\alpha$ lifts formally to $X$ if for every $u \in U$ there is $x \in X$ as above and a homomorphism $\widehat{\beta}$ with $\widehat{\alpha}=\widehat{\beta} \circ \widehat{\pi}$. Clearly, if $\alpha$ lifts, then it lifts formally. A converse is given by the following theorem:

\subsection{Theorem. We assume}

i) $X$ and $Y$ are real-algebraic manifolds and $\pi$ is a morphism.

ii) There is $h \in \mathbb{R}[Y]$ such that $\pi$ is a closed embedding over $Y_{0}:=\{y \in Y \mid$ $h(y) \neq 0\}$.

iii) The function $\bar{h}:=h \circ \alpha \in \mathcal{C}^{\infty}(U)$ is non-zero and locally analytic.

Then $\alpha$ lifts if and only if it lifts formally. Moreover, the lift $\beta$ is unique.

Proof. The map $\beta$ is unique since by iii) the zero-set of $\bar{h}$ is nowhere dense and $\pi$ is injective over $Y_{0}$. For the existence we have to show that $\mathbb{R}[Y] \stackrel{\alpha^{*}}{\longrightarrow} \mathcal{C}^{\infty}(U)$ extends to $\mathbb{R}[X] \stackrel{\beta^{*}}{\longrightarrow} \mathcal{C}^{\infty}(U)$. Because $\alpha$ lifts formally, hence set-theoretically, the image of $\alpha$ is contained in the image of $\pi$. Thus, if $I$ is the kernel of $\mathbb{R}[Y] \stackrel{\pi^{*}}{\rightarrow} \mathbb{R}[X]$, then $\alpha^{*}(I)=0$. Therefore, we obtain a homomorphism $\alpha^{*}: R:=\mathbb{R}[Y] / I \rightarrow \mathcal{C}^{\infty}(U)$ and we can think of $R$ as a subring of $\mathbb{R}[X]$. Because of $i i)$ every $f \in \mathbb{R}[X]$ is of the form $g / h^{N}$, where $g \in R$ and $N \in \mathbb{N}$. Therefore, $\beta^{*}(f)$ exists if and only if $\alpha^{*}(g)$ is divisible by $\bar{h}^{N}$. Because $\bar{h}$ is locally analytic, this can be checked by looking at the Taylor series ( $\mathrm{Mal}$, Thm. 1.1). But for them, divisibility holds since $\alpha$ lifts formally.

\section{Automorphisms of multiplicity free spaCeS}

In this section, we describe the automorphism group of a multiplicity free manifold.

We start by defining a real version of the group scheme $\mathfrak{A}_{\Phi}$. So, let $\Phi=$ $\left(\Lambda, \Delta, \Lambda^{\vee}, \Delta^{\vee}\right)$ be a root system. Recall that $\mathfrak{a}^{*}:=\Lambda \otimes \mathbb{C}$ and $A:=\operatorname{Hom}\left(\Lambda, \mathbb{C}^{\times}\right)$ have the real structures

$$
\overline{\chi \otimes z}=-\chi \otimes \bar{z} \quad \text { and } \quad \bar{a}(\chi)=\overline{a(\chi)}^{-1},
$$

respectively. Then these structures induce a real structure on $\mathfrak{A}_{\Phi}$. Then $\mathfrak{A}_{\Phi}(\mathbb{R})$ is a Lie group scheme over $\mathfrak{s}_{\mathbb{R}}:=\left(\mathfrak{a}^{*} / W\right)(\mathbb{R})$. In the sequel, we need only the part lying over $\mathfrak{a}_{\mathbb{R}}^{*} / W$. Thus, we define

$$
\mathfrak{A}_{\Phi}^{+}:=\mathfrak{A}_{\Phi}(\mathbb{R}) \otimes_{S(\mathbb{R})} \mathfrak{a}_{\mathbb{R}}^{*} / W .
$$

Observe that $\mathfrak{A}_{\Phi}^{+}$is equipped with the structural map $\mathfrak{A}_{\Phi}^{+} \rightarrow \mathfrak{a}_{\mathbb{R}}^{*} / W$ such that each fiber is an (abelian) Lie group. More precisely, there is a unit section $\mathfrak{a}_{\mathbb{R}}^{*} / W \rightarrow \mathfrak{A}_{\Phi}^{+}$ and a multiplication map $\mathfrak{A}_{\Phi}^{+} \times_{\mathfrak{a}_{\mathbb{R}}^{*} / W} \mathfrak{A}_{\Phi}^{+} \rightarrow \mathfrak{A}_{\Phi}^{+}$, both smooth, making each fiber a Lie group. We are going to call such an object a Lie group scheme over $\mathfrak{a}_{\mathbb{R}}^{*} / W$. Let $M$ be a manifold equipped with a smooth map $M \rightarrow \mathfrak{a}_{\mathbb{R}}^{*} / W$. Then an action of $\mathfrak{A}_{\Phi}^{+}$on $M$ over $\mathfrak{a}_{\mathbb{R}}^{*} / W$ is given by a smooth map $\mathfrak{A}_{\Phi}^{+} \times_{\mathfrak{a}_{\mathbb{R}}^{*} / W} M \rightarrow M$ satisfying the usual identities. In particular, each fiber of $\mathfrak{A}_{\Phi}^{+}$over $\mathfrak{a}_{\mathbb{R}}^{*} / W$ (an honest Lie group) acts on the corresponding fiber of $M$ over $\mathfrak{a}_{\mathbb{R}}^{*} / W$ in a smooth fashion.

Next, let $\mathfrak{a}^{0}$ be an affine space with $\mathfrak{a}_{\mathbb{R}}^{*}$ as the group of translations. Assume that $W$ also acts on $\mathfrak{a}^{0}$ in a compatible way. Then, using a point $a \in\left(\mathfrak{a}^{0}\right)^{W}$, one can identify $\mathfrak{a}^{0}$ with $\mathfrak{a}^{*}(\mathbb{R})$ and therefore transport $\mathfrak{A}_{\Phi}^{+}$to a Lie group scheme over $\mathfrak{a}^{0} / W$. This scheme is independent of the choice of $a$ since $\mathfrak{A}_{\Phi}^{+}$is invariant under 
translations by $\left(\mathfrak{a}_{\mathbb{R}}^{*}\right)^{W}$. We call it the Lie group scheme $\mathfrak{A}_{\Phi}^{+}$over $\mathfrak{a}^{0} / W$ (as opposed to $\left.\mathfrak{a}_{\mathbb{R}}^{*} / W\right)$.

9.1. Theorem. Let $M$ be a convex multiplicity free manifold. Then there is a unique root system $\Phi=\Phi_{M}$ with lattice $\Lambda_{M}$ and Weyl group $W_{M}$ and a unique action of the Lie group scheme $\mathfrak{A}_{\Phi}^{+}$over $\mathfrak{s}^{0}:=\mathfrak{a}^{0} / W_{M}$ on $M$ such that

i) the following diagram commutes:

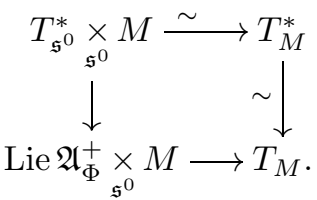

ii) Let $Y=K x \subseteq M$ be an orbit. Then the action of $\mathfrak{A}_{\Phi}^{+}$on $\operatorname{Spec} \mathcal{C}_{Y}^{\infty}(M)^{\mathrm{fin}}$ is saturated.

Proof. First of all, the action is unique since (9.3) prescribes the action of the Lie algebra of $\mathfrak{A}_{\Phi}^{+}$. Also the root system is unique: by construction of $W_{M}$, every root hyperplane $H_{\alpha}$ meets the momentum image $\mathcal{P}$ in some point, say $a$. Let $s$ be its image in $\mathfrak{s}^{0}$. Then in a formal neighborhood of $s$, the length of $\alpha$ is determined by the condition of saturatedness (Theorem 6.9).

For the existence, we construct the action first locally. Let $\mathrm{M}=K \times^{H_{\mathbb{R}}}\left(\mathfrak{h}_{\mathbb{R}}^{\perp} \oplus S\right)$ be a model manifold with corresponding spherical variety $X=G \times{ }^{H} S$. Recall that there is a root system $\Phi=\Phi_{X}$ such that $\mathfrak{A}_{\Phi}$ acts on $T_{X}^{*}$. Let $n \in N_{W}\left(\mathfrak{a}^{*}\right)$ be as in Theorem 3.3 and put $\Phi_{M}:=n(\Phi)$. Then $\mathfrak{A}_{\Phi_{M}}$ is the same as $\mathfrak{A}_{\Phi}$ but with structure morphism twisted by $\mathfrak{a}^{*} / W_{X} \stackrel{n}{\longrightarrow} \mathfrak{a}^{*} / W_{\mathrm{M}}$. Then $\mathfrak{A}_{\Phi_{\mathrm{M}}}$ is acting on $\mathrm{M}^{c}$. Moreover, (3.10) implies that the action is defined over $\mathbb{R}$. Thus, we get an action of $\mathfrak{A}_{\Phi_{M}}^{+}$on M.

Next, let $M$ be any convex multiplicity free manifold and let $K x \subseteq M$ be an orbit. The local cross-section theorem asserts that an open neighborhood of $K x$ is isomorphic to $K \times{ }^{L} \Sigma_{a}$. Here $\Sigma$ is an open neighborhood of $L_{\mathbb{R}} / H_{\mathbb{R}}$ of a model manifold $\mathrm{M}$ for $L_{\mathbb{R}}$ and the subscript $a$ means that the moment map of $\Sigma$ is shifted by $a$. Let $\Phi(a)$ (with $a=\psi(x) \in \mathcal{P}$ ) be the root system attached to $\mathrm{M}$ and $\mathfrak{a}^{0}=a+\mathfrak{a}_{\mathbb{R}}^{*}$. Then the Lie group scheme $\mathfrak{A}_{\Phi(a)}^{+}$on $\mathfrak{a}^{0} / W_{\mathrm{M}}$ acts on $\Sigma_{a}$, hence on a neighborhood of $K x$ in $M$.

Now we claim that it suffices to show that there is a root system $\Phi_{M}$ with $\Phi(a)=\left(\Phi_{M}\right)_{a}$ for all $a \in \mathcal{P}$. Indeed, then the Lie group scheme $\mathfrak{A}_{\Phi_{M}}^{+}$is, near $a$, isomorphic to $\mathfrak{A}_{\Phi(a)}^{+}$(see (15.13)$)$. Moreover, by uniqueness, (9.3), the local actions glue to a global action of $\mathfrak{A}_{\Phi_{M}}^{+}$.

To prove the existence of $\Phi_{M}$ we first remark that the local root systems $\Phi(a)$ have the coherence property

$$
\Phi(b)=\Phi(a)_{b}
$$

for all $b \in \mathcal{P}$ in a neighborhood of $a$. Indeed, this follows from Theorem 6.9 since the action of both $\mathfrak{A}_{\Phi(b)}$ and $\mathfrak{A}_{\Phi(a)_{b}}$ on a formal neighborhood of $K y($ with $\psi(y)=b)$ is saturated.

Let $\Phi_{\max }$ be the maximal root system with Weyl group $W_{M}$ and let $\Sigma_{\max }$ be a set of simple roots with respect to the chamber $\mathfrak{a}^{+}$. We call a simple root $\alpha \in \Sigma_{\max }$ critical if it is not primitive in $\Lambda_{M}$. Critical roots have two important properties. 
First, criticality is just a property of the triple $\left(\alpha, \alpha^{\vee}, \Lambda_{M}\right)$. In particular, $\alpha$ is critical in $\Sigma$ if and only if it is critical in any subset of $\Sigma$ containing $\alpha$. Second, a critical simple root is not in the same $W_{M}$-orbit of any other simple root. This follows from the classification in Lemma 6.6. Thus we can replace any set of critical roots by their halves and still obtain a root system.

We apply this as follows: let $H_{\alpha}$ be the reflection hyperplane for a critical root $\alpha$. Since $\mathcal{P}$ is convex, the intersection $H_{\alpha} \cap \mathcal{P}$ is convex, hence connected. Thus the simple reflection $s_{\alpha}$ appears in the Weyl group of $\Phi(a)$ for all $a \in H_{\alpha} \cap \mathcal{P}$. The coherence property (9.4) implies therefore that there is $n_{\alpha} \in\left\{1, \frac{1}{2}\right\}$ such that $n_{\alpha} \alpha$ is a root of $\Phi(a)$ for all $a \in H_{\alpha} \cap \mathcal{P}$. If $\alpha$ is not critical, we define $n_{\alpha}=1$. Now let $\Phi_{M}$ be the root system with simple roots $\Sigma_{M}:=\left\{n_{\alpha} \alpha \mid \alpha \in \Sigma\right\}$.

It remains to show that $\Phi(a)=\left(\Phi_{M}\right)_{a}$ for all $a \in \mathcal{P}$. So, let $\alpha$ be a simple root of $\Phi(a)$. If $\alpha$ is not critical, then $n_{\alpha}=1$ and $\alpha$ is a simple root of $\Phi_{M}$. Otherwise, $\alpha$ is a simple root of $\Phi_{M}$ by construction. Thus $\Phi(a) \subseteq\left(\Phi_{M}\right)_{a}$. On the other hand, if $\alpha$ is a simple root of $\left(\Phi_{M}\right)_{a}$, then $s_{\alpha}$ is in the Weyl group of $\Phi(a)$ (Lemma 4.5). Thus $\alpha$ is a positive multiple of a simple root $\alpha^{\prime}$ of $\Phi(a)$. Since $\alpha^{\prime} \in\left(\Phi_{M}\right)_{a}$ this implies $\alpha=\alpha^{\prime} \in \Phi(a)$.

Now we are finally in the position to determine the automorphism group of a convex multiplicity free manifold. For the statement, the smooth invariant moment $\operatorname{map} M \stackrel{\psi}{\rightarrow} \mathcal{P} \rightarrow \mathcal{P} / W_{M}$ is denoted by $\psi_{\infty}$.

9.2. Theorem. Let $M$ be a convex multiplicity free manifold with momentum image $\mathcal{P}$. Let $\varphi: M \rightarrow M$ be a diffeomorphism. Then the following are equivalent:

i) $\varphi$ is $K$-equivariant and the momentum map is $\varphi$-invariant, i.e., $m \circ \varphi=m$.

ii) There is a smooth section $u: \mathcal{P} / W_{M} \rightarrow \mathfrak{A}_{\Phi_{M}}^{+}$which induces $\varphi$, i.e. with $\varphi(x)=u\left(\psi_{\infty}(x)\right) x$ for all $x \in M$.

Moreover, in case these conditions hold, the following are equivalent:

iii) The map $\varphi$ is a Hamiltonian automorphism of $M$; i.e., $\varphi$ preserves additionally the symplectic structure of $M$.

iv) The image of the section $u$ is a Lagrangian submanifold of $\mathfrak{A}_{\Phi_{M}}^{+}$.

Proof. Let $u$ be a section of $\mathfrak{A}^{+}:=\mathfrak{A}_{\Phi_{M}}^{+}$and let $\varphi$ be the automorphism of $M$ induced by $u$. Then $\varphi$ is clearly smooth and $K$-equivariant. Moreover, Theorem 7.1 implies that the moment map is $\varphi$-invariant.

Conversely, let $\varphi$ be a smooth $K$-equivariant automorphism preserving the moment map. The (continuous) map $\psi: M \rightarrow \mathfrak{t}^{+}$factors through $m$; hence it is $\varphi$-invariant as well. It follows that the smooth invariant moment map $\psi_{\infty}: M \rightarrow$ $\mathfrak{a}^{0} / W_{M}$ is also $\varphi$-invariant. In particular, $\varphi$ maps each $K$-orbit into itself.

The Lie group scheme $\mathfrak{A}^{+}$acts freely on the dense open subset lying over the interior of $\mathcal{P} / W_{M}$. This implies that the section $u$ of $\mathfrak{A}^{+}$is unique if it exists. In particular, existence is a local problem over $\mathcal{P} / W_{M}$. Let $K x \subseteq M$ be an orbit. Since the $\mathfrak{A}^{+}$-action is compatible with the cross-section theorem (see the proof of Theorem 9.1), we may assume that $m(x)=0$ and that $M$ is a convex open neighborhood of $K / H_{\mathbb{R}}$, a model variety $\mathrm{M}$. 
Now consider the following diagram:

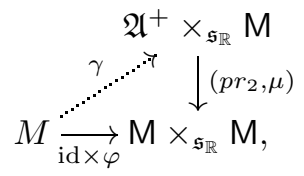

where $\mu$ denotes the $\mathfrak{A}^{+}$-action. Since this action is generically free, the vertical arrow is a closed embedding outside of some hypersurface $h=0$. More precisely, $h$ is the pull-back of a polynomial function $h_{0}$ on $\mathfrak{s}_{\mathbb{R}}$. Thus, since $\psi_{\infty}: M \rightarrow \mathfrak{s}_{\mathbb{R}}$ is real analytic, also the pull-back of $h$ to $M$ is real analytic. Therefore, Theorem 8.3 implies that $\gamma$ exists if and only if it exists formally. For this to check, let $Y=$ $K y \subseteq M$ be a second orbit. Then $\varphi$ induces an automorphism $\widehat{\varphi}$ of

$$
\mathcal{C}_{Y}^{\infty}(M)^{\mathrm{fin}}=\mathbb{R}\left[M_{0}\right] \otimes_{\mathbb{R}\left[M_{0}\right]^{K}} \mathbb{R} \llbracket M_{0} \rrbracket^{K}
$$

(Lemma 8.2). Moreover, the automorphism of the right-hand side preserves both the moment map and the invariant moment map. Thus, Theorem 7.4 implies that $\widehat{\varphi}$ is induced by a formal section $\widehat{u}$ of $\mathfrak{A}^{+}$. But this means exactly that $\gamma$ exists formally. We conclude the existence of the smooth map $\gamma$.

Now consider the composition $\delta: M \stackrel{\beta}{\rightarrow} \mathfrak{A}^{+} \times_{\mathfrak{S}_{\mathbb{R}}} \mathrm{M} \stackrel{p r_{1}}{\longrightarrow} \mathfrak{A}^{+}$and the diagram

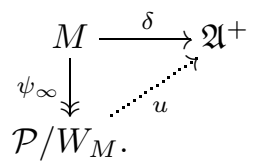

Then $u$ exists since $\delta$ is $K$-invariant and $\psi_{\infty}$ is the smooth quotient map by $K$. Clearly, $u$ is the section whose existence has been asserted.

Finally, the equivalence of iii) and iv) follows from equation (7.8).

Example. A smallest example where $\mathfrak{A}_{\Phi_{M}}^{+}$has non-connected fibers is $M=T_{S_{2}}^{*}$, the cotangent bundle of the 2-sphere, with $K=S O(3)$ acting. Here $A_{\mathbb{R}}^{\Phi_{M}}=\{ \pm 1\}$ and -1 corresponds to the automorphism induced by the antipode map. For a compact example, one can take $M=\mathbf{P}^{1}(\mathbb{C}) \times \mathbf{P}^{1}(\mathbb{C})$ with $K=S U(2)$ acting. Then -1 induces the flip of the two factors.

In analogy with Proposition 4.6, we state a couple of equivalent formulations of what a section of $\mathfrak{A}^{+}$is. First, recall the universal property (5.5) of $\mathfrak{A}_{\Phi}$. For $Y=\mathfrak{A}_{\Phi}$ the identity map corresponds to a $\Phi$-morphism $\mathfrak{A}_{\Phi} \times_{\mathfrak{s}} \mathfrak{a}^{*} \rightarrow A$. Because $\mathfrak{a}^{+} \rightarrow \mathfrak{s}_{\mathbb{R}}$ is injective, we obtain a map

$$
\Psi: \mathfrak{A}_{\Phi}^{+} \stackrel{\text { homeom. }}{\longrightarrow} \mathfrak{A}_{\Phi}^{+} \times_{\mathfrak{s}_{\mathbb{R}}} \mathfrak{a}^{+} \longrightarrow A_{\mathbb{R}}
$$

For example, for the group scheme (5.1) this is the map

$$
\left(\left(\begin{array}{cc}
a & s b \\
b & a
\end{array}\right), s\right) \mapsto\left(\begin{array}{cc}
a & -\sqrt{-s} b \\
\sqrt{-s} b & a
\end{array}\right)
$$

(Observe, that $s \in(i \mathbb{R})^{2}=\mathbb{R}_{\leq 0}$.) The map $\Psi$ fits into the commutative diagram

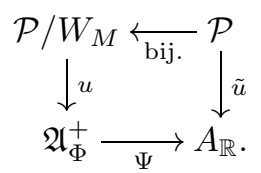


Since $W_{M}$ does not act on $\mathcal{P}$ we need the following:

9.3. Definition. Let $\tilde{u}: \mathcal{P} \rightarrow A_{\mathbb{R}}$ be a smooth map.

i) $\tilde{u}$ is $\Phi$-equivariant if for every $a \in \mathcal{P}$ there is a $\left(W_{M}\right)_{a}$-invariant open neighborhood $U \subseteq \mathfrak{a}_{\mathbb{R}}^{*}$ and a $\Phi_{a}$-equivariant smooth map $U \rightarrow A_{\mathbb{R}}$ which coincides with $\tilde{u}$ on $U \cap \mathcal{P}$.

ii) $\tilde{u}$ is formally $\Phi$-equivariant if for every $a \in \mathcal{P}$ the morphism $\widehat{\mathfrak{a}}_{a}^{*} \rightarrow A$ is $\Phi_{a}$-equivariant. Here, $\widehat{\mathfrak{a}}_{a}^{*}$ is the formal neighborhood of $a$ in $\mathfrak{a}_{\mathbb{R}}^{*}$.

9.4. Proposition. The assignment $u \mapsto \tilde{u}$ induces a bijection between

i) smooth sections of $\mathfrak{A}_{\Phi}^{+}$over $\mathcal{P}$,

ii) $\Phi$-equivariant smooth maps $\mathcal{P} \rightarrow A_{\mathbb{R}}$, and

iii) formally $\Phi$-equivariant smooth maps $\mathcal{P} \rightarrow A_{\mathbb{R}}$.

Proof. Let $u: \mathcal{P} / W \rightarrow \mathfrak{A}^{+}$be a smooth section. Then $\tilde{u}$ is a composition of smooth maps:

$$
\tilde{u}: \mathcal{P} \longrightarrow \mathfrak{A}_{\Phi}^{+} \times_{\mathfrak{s}_{\mathbb{R}}} \mathfrak{a}^{+} \longrightarrow A_{\mathbb{R}}
$$

and therefore smooth. It is (formally) $\Phi$-equivariant since the right arrow is.

Conversely, let $\tilde{u}: \mathcal{P} \rightarrow A_{\mathbb{R}}$ be formally $\Phi$-equivariant. To construct $u$, consider the smooth map

$$
\mathfrak{A}_{\Phi}^{+} \times \mathfrak{s}_{\mathbb{R}} \mathfrak{a}^{+} \longrightarrow A_{\mathbb{R}} \times \mathfrak{a}_{\mathbb{R}}^{*}
$$

Passing to the quotient by $W$ we get a smooth map $\mathfrak{A}_{\Phi}^{+} \rightarrow A_{\mathbb{R}} \times{ }^{W} \mathfrak{a}_{\mathbb{R}}^{*}$, which is an isomorphism over the regular part of $\mathfrak{a}_{\mathbb{R}}^{*}$. The map $\tilde{u}$ gives rise to a map $\bar{u}: \mathcal{P} / W \rightarrow A_{\mathbb{R}} \times{ }^{W} \mathfrak{a}_{\mathbb{R}}^{*}$. Thus, $u$ fits into the following diagram:

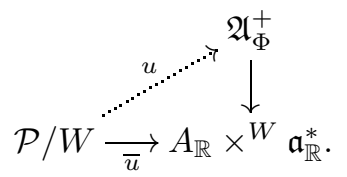

The lift $u$ exists formally because of the universal property of $\mathfrak{A}_{\Phi}$. Thus $u$ exists uniquely by Theorem 8.3 .

\section{Cohomology COMPutation}

For a root datum $\Phi=\left(\Lambda, \Delta, \Lambda^{\vee}, \Delta^{\vee}\right)$ with Weyl group $W$ and a convex subset $\mathcal{P} \subseteq \mathfrak{a}^{*}=\Lambda \otimes_{\mathbb{Z}} i \mathbb{R}$ with non-empty interior, let $\mathcal{L}_{\mathcal{P}}^{\Phi}$ be the sheaf of Lagrangian sections of $\mathfrak{A}_{\Phi}^{+}$over $\mathcal{P} / W$. Then Theorem 9.2 can be rephrased as $\operatorname{Aut}\left(M_{U}\right)=$ $\mathcal{L}_{\mathcal{P}}^{\Phi_{M}}(U)$, where $U \subseteq \mathcal{P} / W_{M}$ is open and $M_{U}=\psi_{\infty}^{-1}(U)$. Thus, in view of Theorem 2.4, the Delzant conjecture follows from the case $i=1$ of the following theorem.

10.1. Theorem. Let $\Phi$ be a root datum and $\mathcal{P} \subseteq \mathfrak{a}^{+}$a locally polyhedral convex subset with non-empty interior. Then $H^{i}\left(\mathcal{P} / W, \mathcal{L}_{\mathcal{P}}^{\Phi}\right)=0$ for $i \geq 1$.

Proof. Let $R=\langle\Delta\rangle \subseteq \Lambda$ be the root lattice and put $\Lambda_{0}:=R \oplus \Lambda^{W} \subseteq \Lambda$. This sublattice is of finite index in $\Lambda$ and gives rise to a new root datum $\Phi_{0}$. Then, according to section 5 , the finite group $E:=\operatorname{Hom}\left(\Lambda / \Lambda_{0}, \mathbb{C}^{\times}\right)$is acting freely on $\mathfrak{A}_{\Phi}^{+}$ with quotient $\mathfrak{A}_{\Phi}^{+} / E=\mathfrak{A}_{\Phi_{0}}^{+}$. Since Lagrangian sections are mapped to Lagrangian sections one gets a short exact sequence

$$
1 \rightarrow E_{\mathcal{P} / W} \rightarrow \mathcal{L}_{\mathcal{P}}^{\Phi} \rightarrow \mathcal{L}_{\mathcal{P}}^{\Phi_{0}} \rightarrow 1
$$


where $E_{\mathcal{P} / W}$ is the constant sheaf with fiber $E$. Since $\mathcal{P}$ is convex, hence contractible, we have $H^{i}\left(\mathcal{P} / W, E_{\mathcal{P} / W}\right)=0$ for $i \geq 1$. Thus it suffices to show that $\mathcal{L}_{\mathcal{P}}^{\Phi_{0}}$ has no higher cohomology.

Replacing $\Phi$ by $\Phi_{0}$, we may assume from now on that the root lattice $R$ is a direct summand of $\Lambda$. Since the root lattice of $\Delta_{a}$ is a direct summand of $R$ this implies that all fibers of $\mathfrak{A}_{\Phi}^{+} \rightarrow \mathfrak{a}_{\mathbb{R}}^{*} / W$ are connected (see (5.10) ). Hence, the exponential map

$$
\exp : T_{\mathfrak{a}_{\mathbb{R}}^{*} / W}^{*}=\operatorname{Lie} \mathfrak{A}_{\Phi}^{+} \rightarrow \mathfrak{A}_{\Phi}^{+}
$$

is surjective. Being a local isomorphism, locally every section of $\mathfrak{A}_{\Phi}^{+}$is of the form $\exp \omega$, where $\omega$ is a 1 -form on $\mathfrak{a}_{\mathbb{R}}^{*} / W$. Moreover, $\exp \omega$ is Lagrangian if and only if $\omega$ is closed. The convexity of $\mathcal{P}$ implies that then, again locally, $\omega=d f$ for some smooth function $f$ on $\mathcal{P} / W$. Thus we have shown that there is a short exact sequence

$$
0 \rightarrow \mathcal{K} \longrightarrow \mathcal{C}_{\mathcal{P} / W}^{\infty} \stackrel{\exp d f}{\longrightarrow} \mathcal{L}_{\mathcal{P}}^{\Phi} \rightarrow 1 .
$$

The higher cohomology of $\mathcal{C}_{\mathcal{P} / W}^{\infty}$ vanishes. Thus it remains to show that $H^{i}(\mathcal{P} / W, \mathcal{K})=0$ for $i \geq 2$.

The map $\mathcal{P} \rightarrow \mathcal{P} / W$ is a homeomorphism. Therefore, the pull-back $\mathcal{K}^{+}$of $\mathcal{K}$ to $\mathcal{P}$ has the same cohomology. For $U^{+} \subseteq \mathcal{P}$ open the group $\mathcal{K}^{+}\left(U^{+}\right)$consists of smooth functions $f^{+}$on $U^{+}$such that $\exp d f^{+} \equiv 1$. Because $\Lambda^{\vee} \subseteq \mathfrak{a} \cong$ Lie $A$ is the kernel of exp : $\mathfrak{a} \rightarrow A$ the function $f^{+}$must satisfy $d f^{+} \in \Lambda^{\vee}$ over the interior of $\mathcal{P}$. This implies that, at least locally, $f^{+}$is an affine linear function

$$
f^{+}(z)=\langle z \mid \gamma\rangle+c, \quad \text { where } \gamma \in \Lambda^{\vee}, c \in \mathbb{R} .
$$

Now we have to figure out when $f^{+}$represents a smooth function on $\mathcal{P} / W$. By Proposition 4.6 this is the case if the Taylor series of $f^{+}$in any point $a \in \mathcal{P}$ is $W_{a}$-invariant. This translates into

$$
\langle\alpha \mid \gamma\rangle=0 \text { for all } \alpha \in \Delta_{a} .
$$

Thus, we have proved: If $U^{+} \subseteq \mathcal{P}$ is connected, then $\mathcal{K}^{+}\left(U^{+}\right)$is the set of functions $f^{+}(z)=\langle z \mid \gamma\rangle+c$, where $c \in \mathbb{R}, \gamma \in \Lambda^{\vee}$ and $\left\langle\Delta_{a} \mid \gamma\right\rangle=0$ for all $a \in U^{+}$.

The choice of the Weyl chamber $\mathfrak{a}^{+}$determines a system of simple roots $\Sigma \subseteq \Delta$. Then $\Sigma_{a}:=\Sigma \cap \Delta_{a}$ is a set of simple roots for $\Delta_{a}$ for all $a \in \mathfrak{a}^{+}$. Thus every $a \in \mathcal{P}$ has an open neighborhood $U^{+}$such that $\mathcal{K}^{+}\left(U^{+}\right)$is the kernel of

$$
\Lambda^{\vee} \oplus \mathbb{R} \longrightarrow \mathbb{Z}^{\left|\Sigma_{a}\right|}:(\gamma, c) \mapsto(\langle\alpha \mid \gamma\rangle)_{\alpha \in \Sigma_{a}} .
$$

Recall that the root lattice is a direct summand of $\Lambda$. Thus $\Sigma_{a}$ is part of a basis of $\Lambda$, which means that the map (10.6) is surjective. Since $\alpha \in \Sigma_{a}$ can also be rephrased as $a \in H_{\alpha}$, where $H_{\alpha}$ is the reflection hyperplane corresponding to $\alpha$, we get a short exact sequence of sheaves

$$
0 \rightarrow \mathcal{K}^{+} \rightarrow \Lambda_{\mathcal{P}}^{\vee} \oplus \mathbb{R}_{\mathcal{P}} \rightarrow \bigoplus_{\alpha \in \Sigma} \mathbb{Z}_{H_{\alpha} \cap \mathcal{P}} \rightarrow 0
$$

Here, $\mathbb{Z}_{H_{\alpha} \cap \mathcal{P}}$ is the sheaf which is constant $\mathbb{Z}$ on $H_{\alpha} \cap \mathcal{P}$ and zero outside. Also $H_{\alpha} \cap \mathcal{P}$ is convex for all $\alpha$, which implies that $H^{i}\left(\mathcal{P}, \mathbb{Z}_{H_{\alpha} \cap \mathcal{P}}\right)=0$ for all $i \geq 1$. From this the desired vanishing for $\mathcal{K}^{+}$follows. 
Now we are able to prove the Delzant Conjecture:

10.2. Theorem. A convex multiplicity free manifold is uniquely characterized by its momentum polyhedron together with its principal isotropy group.

Proof. The proof is now standard. Let $M$ and $M^{\prime}$ be two convex multiplicity free manifolds having the same moment polytope $\mathcal{P}$ and the same principal isotropy group $L_{0}$. By Theorem 2.4 there is a covering $U_{\nu}$ of $\mathcal{P}$ by convex open subsets and isomorphisms $\varphi_{\nu}: M_{U_{\nu}} \stackrel{\sim}{\rightarrow} M_{U_{\nu}}^{\prime}$. Put $U_{\mu \nu}:=U_{\mu} \cap U_{\nu}, \tilde{U}_{\nu}:=U_{\nu} / W_{M}$, and $\tilde{U}_{\mu \nu}:=$ $U_{\mu \nu} / W_{M}$. Then Theorem 9.2 implies that the automorphism $\varphi_{\mu}^{-1} \varphi_{\nu}$ of $M_{U_{\mu \nu}}$ is induced by a unique section $\alpha_{\mu \nu}$ of $\mathcal{L}_{\mathcal{P}}^{\Phi_{M}}$ over $\tilde{U}_{\mu \nu}$. By uniqueness, these sections satisfy the cocycle condition $\alpha_{\lambda \nu}=\alpha_{\lambda \mu} \alpha_{\mu \nu}$ on $\tilde{U}_{\lambda} \cap \tilde{U}_{\mu} \cap \tilde{U}_{\nu}$. The covering $\tilde{U}_{\nu}$ of $\mathcal{P} / W_{M}$ is $\mathcal{L}_{\mathcal{P}}^{\Phi}$-acyclic by Theorem 10.1 Hence the vanishing of $H^{1}\left(\mathcal{P} / W_{M}, \mathcal{L}_{\mathcal{P}}^{\Phi^{M}}\right)$ implies that there are sections $\beta_{\nu}$ of $\mathcal{L}_{\mathcal{P}}^{\Phi_{M}}$ over $\tilde{U}_{\nu}$ with $\alpha_{\mu \nu}=\beta_{\mu}^{-1} \beta_{\nu}$ on $\tilde{U}_{\mu \nu}$. Thus replacing the automorphisms $\varphi_{\nu}$ by $\varphi_{\nu} \circ \beta_{\nu}$ one obtains $\varphi_{\mu}=\varphi_{\nu}$ over $U_{\mu \nu}$. Therefore, the $\varphi_{\nu}$ glue to a global isomorphism $\varphi: M \stackrel{\sim}{\rightarrow} M^{\prime}$.

\section{The ClASSIFICATION OF MULTIPLICITY FREE MANIFOLDS}

Now we are able to complete the classification of convex multiplicity free manifolds. For this, we establish the following notation. Let $\Phi=\left(\Lambda, \Delta, \Lambda^{\vee}, \Delta^{\vee}\right)$ be a root datum. After choosing a system $\Delta^{+}$of positive roots, these data define the usual triple $T \subseteq B \subseteq G$, where $G$ is a connected complex reductive group, $B$ is a Borel subgroup, and $T$ is a maximal torus.

11.1. Definition. Let $\Lambda_{0} \subseteq \Lambda$ be a subgroup. Then a cone $\mathcal{Q} \subseteq \Lambda \otimes \mathbb{R}$ is called multiplicity free for $\left(\Phi, \Delta^{+}, \Lambda_{0}\right)$ if there is a smooth affine spherical $G$-variety $X$ whose weight monoid $\Xi_{X}$ satisfies

$$
\begin{aligned}
\mathcal{Q} & =\text { convex cone generated by } \Xi_{X}, \\
\Lambda_{0} & =\text { abelian group generated by } \Xi_{X} .
\end{aligned}
$$

Recall also the notion of a tangent cone of a subset $\mathcal{Q}$ of a real vector space in $a \in \mathcal{Q}$ : it is the convex cone generated by the set $\mathcal{Q}-a$. The advertised classification is:

11.2. Theorem. Let $K$ be a compact connected Lie group with root datum $\Phi$ and a choice of positive roots $\Delta^{+}$. Then there is a bijection between isomorphism classes of convex multiplicity free $K$-manifolds and pairs $\left(\mathcal{Q}, \Lambda_{0}\right)$ such that

i) $\Lambda_{0}$ is a subgroup of $\Lambda$ and $\mathcal{Q}$ is a locally polyhedral convex subset of the Weyl chamber determined by $\Delta^{+}$and

ii) the tangent cone of every $a \in \mathcal{Q}$ is multiplicity free for the triple $\left(\Phi_{a}, \Delta_{a}^{+}, \Lambda_{0}\right)$.

Proof. First, note that we replaced the moment polytope $\mathcal{P}$ sitting in $\Lambda \otimes i \mathbb{R}$ by $\mathcal{Q}=\frac{1}{i} \mathcal{P} \subseteq \Lambda \otimes \mathbb{R}$. The only thing left to prove is the existence of $M$ for any pair $\left(\mathcal{Q}, \Lambda_{0}\right)$ satisfying the two conditions. By definition, there is an open convex cover $U_{\nu}$ of $\mathcal{P}$ and convex multiplicity free manifolds $M_{\nu}$ with invariants $\left(\frac{1}{i} U_{\nu}, \Lambda_{0}\right)$. Moreover, by Delzant's conjecture, these $M_{\nu}$ are all isomorphic over the intersection $U_{\mu} \cap U_{\nu}$. In particular, these manifolds yield a well-defined system of local root systems $\Phi(a), a \in \mathcal{P}$. Now observe that in the construction of the global root 
system $\Phi_{M}$, only the two coherence properties (4.5) and (9.4) entered. Thus, there is a root system $\Phi$ with $\Phi(a)=\Phi_{a}$ for all $a \in \mathcal{P}$. Let $W$ be its Weyl group.

The rest is standard. Let $U_{\mu \nu}=U_{\mu} \cap U_{\nu}$ and $U_{\lambda \mu \nu}=U_{\lambda} \cap U_{\mu} \cap U_{\nu}$. The isomorphisms $\varphi_{\mu \nu}:\left(M_{\nu}\right)_{U_{\mu \nu}} \stackrel{\sim}{\rightarrow}\left(M_{\mu}\right)_{U_{\mu \nu}}$ induce an automorphism $\varphi_{\lambda \nu}^{-1} \varphi_{\lambda \mu} \varphi_{\mu \nu}$ of $\left(M_{\nu}\right)_{U_{\lambda \mu \nu}}$ and therefore a section $\alpha_{\lambda \mu \nu} \in \Gamma\left(U_{\lambda \mu \nu} / W, \mathcal{L}_{\mathcal{P}}^{\Phi}\right)$. Using the commutativity of $\mathcal{L}_{\mathcal{P}}^{\Phi}$ one verifies easily that these sections satisfy the cocycle condition

$$
\alpha_{\kappa \lambda \mu} \alpha_{\kappa \lambda \nu}^{-1} \alpha_{\kappa \mu \nu} \alpha_{\lambda \mu \nu}^{-1}=1
$$

on $\left(U_{\kappa} \cap U_{\lambda} \cap U_{\mu} \cap U_{\nu}\right) / W$. The cover $U_{\nu} / W$ is $\mathcal{L}_{\mathcal{Q}}^{\Phi}$-acyclic. Hence the vanishing of $H^{2}\left(\mathcal{Q} / W, \mathcal{L}_{\mathcal{Q}}^{\Phi}\right)$ implies the existence of sections $\beta_{\mu \nu} \in \Gamma\left(U_{\mu \nu}, \mathcal{L}_{\mathcal{Q}}^{\Phi}\right)$ with $\alpha_{\lambda \mu \nu}=$ $\beta_{\lambda \mu} \beta_{\lambda \nu}^{-1} \beta_{\mu \nu}$. Thus replacing the isomorphisms $\varphi_{\mu \nu}$ by $\varphi_{\mu \nu} \beta_{\mu \nu}^{-1}$ yields $\alpha_{\lambda \mu \nu}=1$, i.e., $\varphi_{\lambda \mu}=\varphi_{\lambda \mu} \varphi_{\mu \nu}$. This means that the manifolds $M_{\nu}$ unambiguously glue to a space $M$ over $\mathcal{P}$.

It is not necessary to check condition ii) for all $a \in \mathcal{P}$. For example, condition ii) is automatically satisfied for interior points of $\mathcal{P}$. More generally, let $\mathcal{F} \subseteq \mathcal{P}$ be a face. Then $\Phi_{a}, \Delta_{a}^{+}$and the tangent cone of $\mathcal{P}$ in $a$ are the same for all points $a$ in the relative interior $\mathcal{F}^{0}$ of $\mathcal{F}$. So it suffices to check condition $i i$ ) for one point in $\mathcal{F}^{0}$, where $\mathcal{F}$ runs through all faces of $\mathcal{P}$. Moreover, ii) is almost by construction an open condition. Thus it suffices to check only the minimal faces. In particular, if $\mathcal{P}$ is compact, then it suffices to check ii) for the vertices alone. To do this, one only needs to consider spherical varieties whose cone $\mathcal{Q}$ is pointed. This way, we recover Delzant's theorem [De1]:

11.3. Corollary. Let $K=T_{\mathbb{R}}$ be a torus. Then the compact multiplicity free $T_{\mathbb{R}}$ manifolds $M$ with $T_{\mathbb{R}}$ acting effectively are classified by compact simple regular polytopes $\mathcal{P}$, i.e., by those polytopes such that the tangent cone of $\mathcal{P}$ in any vertex is spanned by a basis of $X\left(T_{\mathbb{R}}\right)$.

Proof. Let $X$ be a smooth affine spherical $T$-variety with $T$. The convex cone $\mathcal{Q}$ is pointed if and only if $H=T$. Thus $X=S=\mathbb{C}^{r}$ is a vector space on which $T$ acts with characters $\chi_{1}, \ldots, \chi_{r}$. Sphericality of $X$ means that the $\chi_{\nu}$ are linearly independent while effectivity of the action implies that $X(T)$ is generated by the $\chi_{\nu}$. Thus, $\mathcal{Q}$ is spanned by a basis of $X(T)$.

We can also easily recover the classification of Iglésias [Igl]:

11.4. Corollary. Let $M$ be a compact multiplicity free $G=S U(2)$-manifold.Then there are the following possibilities for $(\mathcal{P}, \Lambda)$ :

i) $\mathcal{P}=\{x\} \subseteq \mathbb{R}_{\geq 0}$ and $\Lambda=0$. The corresponding manifold $M$ is a coadjoint orbit, i.e., either a point $(x=0)$ or a $\mathbf{P}_{\mathbb{C}}^{1}(x>0)$.

ii) $\mathcal{P}=[0, y] \subseteq \mathbb{R}_{\geq 0}$ with $y>0$ and $\Lambda=d \mathbb{Z}$ with $d \in\{1,2,4\}$. The corresponding manifold $M$ is either $\mathbf{P}_{\mathbb{C}}^{2} \cong \mathbf{P}\left(\mathbb{C}^{2} \oplus \mathbb{C}\right)(d=1), \mathbf{P}_{\mathbb{C}}^{1} \times \mathbf{P}_{\mathbb{C}}^{1}(d=2)$, or $\mathbf{P}_{\mathbb{C}}^{2} \cong \mathbf{P}(\mathfrak{s l}(2, \mathbb{C}))(d=4)$.

iii) $\mathcal{P}=[x, y] \subseteq \mathbb{R}_{\geq 0}$ with $0<x<y$ and $\Lambda=d \mathbb{Z}$ with $d \in \mathbb{Z}_{>0}$. The corresponding manifold $M$ is a Hirzebruch surface of degree $d$.

Here we identified the weight lattice with $\mathbb{Z}$ and $\mathfrak{t}^{+}$with $\mathbb{R}_{\geq 0}$.

Proof. We are either in case $i$ ) or $\mathcal{P}=[x, y], 0 \leq x<y$, is a closed interval. Assume the latter. Then $\Lambda=d \mathbb{Z}$ for some $d \in \mathbb{Z}_{>0}$. 
If $x>0$, then all root systems $\Phi_{a}$ are empty; i.e., $(\mathcal{P}, \Lambda)$ is a simple regular polytope. This is always the case and leads to iii).

Now assume $\mathcal{P}=[0, y]$. Then the local model $X$ over $a=0$ is a smooth affine spherical $S L(2, \mathbb{C})$-variety. These are easily classified: $V=0$ and $H=\mathbb{C}^{\times}$or $H=N_{H}\left(\mathbb{C}^{\times}\right)$, leading to the cases $d=2$ or $d=4$ in $\left.i i\right)$, or $H=G$ and $V=\mathbb{C}^{2}$ corresponding to $d=1$.

In general, it is possible but quite tedious to give a full list of multiplicity free cones for any given triple $\left(\Phi, \Delta^{+}, \Lambda_{0}\right)$. Delzant De2 has effectively done this in the rank-2 case. In general, one can use the classification of smooth affine spherical varieties in KVS.

On the other hand, in the past decade we have seen significant progress on the combinatorial structure of multiplicity free cones, especially through Luna's approach Lun for classifying all spherical varieties. For example, it was these methods which enabled Losev to prove the "Knop Conjecture", Theorem 2.7. We refer the reader to Losev's work $\mathrm{Los}$ for more information on these matters.

Another benefit of Theorem 11.2 is that the construction of multiplicity free manifolds is now a purely local process. For example, consider the natural action of a torus $T=U(1)^{n}$ on $X=\mathbb{C}^{n}$. Then $X$ is multiplicity free, with $\mathcal{P}=\mathbb{R}_{>0}^{n}$ and $\Lambda=\mathbb{Z}^{n}$. Let $x_{i}$ be the coordinates of $\mathbb{R}^{n}$, choose $\varepsilon>0$ and "cut off" a corner of size $\varepsilon$ off $\mathcal{P}$. More precisely, let

$$
\mathcal{P}_{\varepsilon}:=\left\{\left(x_{i}\right) \in \mathcal{P} \mid x_{1}+\ldots+x_{n} \geq \varepsilon\right\} .
$$

It is easily verified that $\left(\mathcal{P}_{\varepsilon}, \Lambda\right)$ is still a simple regular polytope, so corresponds to a Hamiltonian manifold $X_{\varepsilon}$ (in fact, $X_{\varepsilon}$ is simply the blowup of $\mathbb{C}^{n}$ in the origin). Now, Theorem 11.2 allows us to perform this process locally: let $M$ be a multiplicity free manifold with data $\mathcal{P}$ and $\Lambda$. Let $\mathcal{F}$ be the smallest face of $\mathfrak{t}^{+}$containing $\mathcal{P}$ and let $a \in \mathcal{P}$ be a vertex lying in the interior of $\mathcal{F}$. Then $(\mathcal{P}, \Lambda)$ is a simple regular polytope near $a$, i.e., looks like the example above. Thus, we can cut off a small enough corner of $\mathcal{P}$ at $a$ to get a polytope $\mathcal{P}_{\varepsilon}$ which corresponds to a manifold $M_{\varepsilon}$. This construction is not new since it corresponds in fact to (a special case) of Lerman's symplectic cuts Ler. More complicated "surgeries" even at non-toroidal vertices are imaginable, though.

\section{ACKNOWLEDGMENT}

The author would like to thank Yael Karshon, Eugen Lerman, Reyer Sjamaar, Sue Tolman, and Chris Woodward for very fruitful discussions. This is especially true for the analytical and topological side of this paper. At the time of its conception, the author was supported by grants from the NSF and the NSA.

\section{REFERENCES}

[AtMa] Atiyah, M.; Macdonald, I., Introduction to commutative algebra, Addison-Wesley Publishing Co., Reading, Mass.-London-Don Mills, Ont. (1969). MR.0242802 (39:4129)

[BiMi] Bierstone, E.; Milman, P., Composite differentiable functions, Ann. of Math. (2) 116 (1982), 541-558. MR678480 (84a:58016)

[Bri] Brion, M., Sur l'image de l'application moment in: Séminaire d'algèbre Paul Dubreil et Marie-Paule Malliavin (Paris, 1986) Lecture Notes in Math. 1296, Springer, Berlin (1987), 177-192. MR932055 (89i:32062) 
[BrTi] Bruhat, F.; Tits, J., Groupes réductifs sur un corps local. II. Schémas en groupes. Existence d'une donnée radicielle valuée, Publ. Math., Inst. Hautes Études Sci. 60 (1984), 5-184. MR0756316 (86c:20042)

[Cam] Camus, R., Variétés sphériques affines lisses, Thèse de doctorat (Université J. Fourier) (2001).

[De1] Delzant, T., Hamiltoniens périodiques et images convexes de l'application moment, Bull. Soc. Math. France 116 (1988), 315-339. MR984900 (90b:58069)

[De2] Classification des actions hamiltoniennes complètement intégrables de rang deux, Ann. Global Anal. Geom. 8 (1990), 87-112. MR.1075241 (92f:58078)

[GSj] Guillemin, V.; Sjamaar, R., Convexity properties of Hamiltonian group actions, CRM Monograph Series 26, American Mathematical Society, Providence, RI (2005). MR2175783(2007c:53119)

[GS] Guillemin, V.; Sternberg, S., Multiplicity-free spaces, J. Diff. Geom. 19 (1984), 31-56. MR739781 (85h:58071)

[Igl] Iglésias, P., Les $\mathrm{SO}(3)$-variétés symplectiques et leur classification en dimension 4, Bull. Soc. Math. France 119 (1991), 371-396. MR1125672 (92i:57023)

[Kac] Kac, V., Infinite dimensional Lie algebras, 3rd ed. Cambridge University Press, Cambridge (1990). MR1104219 (92k:17038)

[KaLe] Karshon, Y.; Lerman, E., The centralizer of invariant functions and division properties of the moment map, Illinois J. Math. 41 (1997), 462-487. MR.1458185 (98e:58077)

[Kir1] Kirwan, F., Convexity properties of the moment mapping. III, Invent. Math. 77 (1984), 547-552. MR759257 (86b:58042b)

[Kir2] Cohomology of quotients in symplectic and algebraic geometry, Mathematical Notes 31, Princeton University Press, Princeton, NJ (1984). MR.766741 (86i:58050)

[Kn1] Knop, F., Weylgruppe und Momentabbildung, Invent. Math. 99 (1990), 1-23. MR.1029388 (91f:14045)

[Kn2] , The asymptotic behavior of invariant collective motion, Invent. Math. 116 (1994), 309-328. MR1253195 (94m:14063)

[Kn3] - Automorphisms, root systems, and compactifications of homogeneous varieties, J. Amer. Math. Soc. 9 (1996), 153-174. MR1311823 (96c:14037)

[Kn4] - Towards a classification of multiplicity free manifolds, Handout for the conference "Journées Hamiltoniennes" in Grenoble (Nov. 29-30, 1997), 8 pages.

[Kn5] W Weyl groups of Hamiltonian manifolds, I, Preprint (1997), 33 pages, dg-ga/9712010.

[Kn6] Convexity of Hamiltonian manifolds, J. Lie Theory 12 (2002), 571-582, math/0112144. MR1923787 (2003j:53131)

[KVS] Knop, F.; Van Steirteghem, B., Classification of smooth affine spherical varieties, Transform. Groups 11 (2006), 495-516, math/0505102. MR2264463 (2007g:14057)

[Ler] Lerman, E., Symplectic cuts, Math. Res. Lett. 2 (1995), 247-258. MR.1338784 (96f:58062)

[LMTW] Lerman, E.; Meinrenken, E.; Tolman, S.; Woodward, Ch., Nonabelian convexity by symplectic cuts, Topology 37 (1998), 245-259, dg-ga/9603015. MR.1489203(99a:58069)

[Los] Losev, I., Proof of the Knop conjecture, Ann. Inst. Fourier (Grenoble) 59 (2009), 11051134, math/0612561. MR2543664 (2010j:14091)

[Lun] Luna, D., Variétés sphériques de type A, Publ. Math. Inst. Hautes Études Sci. 94 (2001), 161-226. MR1896179 (2003f:14056)

[Mag] Magid, A., Equivariant completions and tensor products Group actions and invariant theory (Montreal, PQ, 1988) CMS Conf. Proc. 10, Amer. Math. Soc., Providence, RI (1989), 133-136. MR1021285 (91b:14064)

[Mal] Malgrange, B., Ideals of differentiable functions, Tata Institute of Fundamental Research Studies in Mathematics, No. 3, Oxford University Press, London (1967). MR0212575 $(35: 3446)$

[Mat] Matsumura, H., Commutative ring theory, Cambridge Studies in Advanced Mathematics 8, Cambridge University Press, Cambridge (1986). MR.879273 (88h:13001)

[MiFo] Miščenko, A.; Fomenko, A., A generalized Liouville method for the integration of Hamiltonian systems, Funkcional. Anal. i Priložen. 12 (1978), 46-56, 96. MR0516342 $(58: 24357)$ 
[Sch] Schwarz, G., Smooth functions invariant under the action of a compact Lie group, Topology 14 (1975), 63-68. MR0370643 (51:6870)

[Sja] Sjamaar, R., Convexity properties of the moment map re-examined, Adv. Math. 138 (1998), 46-91, dg-ga/9408001. MR.1645052 (2000a:53148)

[Wo1] Woodward, Ch, The classification of transversal multiplicity-free group actions, Ann. Global Anal. Geom. 14 (1996), 3-42. MR1375064 (97a:58067)

[Wo2] _ Spherical varieties and existence of invariant Kähler structures, Duke Math. J. 93 (1998), 345-377. MR 1625995 (99d:58072)

Department of Mathematics, Universität Erlangen, Bismarckstrasse $1 \frac{1}{2}$, D-91054 ErLangen, Germany 